\title{
Automated Data Acquisition in Construction with Remote Sensing Technologies
}

\author{
Osama Moselhi ${ }^{1}$, Hassan Bardareh ${ }^{2, * \mathbb{D}}$ and Zhenhua Zhu ${ }^{3}$ (D) \\ 1 Centre for Innovation in Construction and Infrastructure Engineering and Management (CICIEM), \\ Department of Building, Civil and Environmental Engineering, Concordia University, \\ 1455 de Maisonneuve Blvd, West, Montreal, QC H3G 1M8, Canada; moselhi@encs.concordia.ca \\ 2 Department of Building, Civil and Environmental Engineering, Concordia University, Sir George Williams \\ Campus, 1455 De Maisonneuve Blvd. W., Montréal, QC H3G 1M8, Canada \\ 3 Department of Civil and Environmental Engineering, University of Wisconsin-Madison, \\ 2258 Engineering Hall, 1415 Engine Drive, Madison, WI 53706, USA; zzhu286@wisc.edu \\ * Correspondence: hassan.bardareh@mail.concordia.ca; Tel.: +1-514-850-7731
}

Received: 7 April 2020; Accepted: 16 April 2020; Published: 20 April 2020

Featured Application: This review article aims to highlight the capabilities and limitations of a wide range of remote sensing (RS) technologies for their possible use for automated data acquisition on construction job sites. More attention is given to integrated RS technologies for the purpose of tracking, localization, and 3D modeling in construction projects. This article is expected to facilitate the use of RS technologies for automating progress reporting, improving safety and productivity in construction.

\begin{abstract}
Near real-time tracking of construction operations and timely progress reporting are essential for effective management of construction projects. This does not only mitigate potential negative impact of schedule delays and cost overruns but also helps to improve safety on site. Such timely tracking circumvents the drawbacks of conventional methods for data acquisition, which are manual, labor-intensive, and not reliable enough for various construction purposes. To address these issues, a wide range of automated site data acquisition, including remote sensing (RS) technologies, has been introduced. This review article describes the capabilities and limitations of various scenarios employing RS enabling technologies for localization, with a focus on multi-sensor data fusion models. In particular, we have considered integration of real-time location systems (RTLSs) including GPS and UWB with other sensing technologies such as RFID, WSN, and digital imaging for their use in construction. This integrated use of technologies, along with information models (e.g., BIM models) is expected to enhance the efficiency of automated site data acquisition. It is also hoped that this review will prompt researchers to investigate fusion-based data capturing and processing.
\end{abstract}

Keywords: automated data acquisition; remote sensing technologies; automated progress reporting; data fusion; tracking resources

\section{Introduction}

Having timely access to accurate and reliable onsite information is vital for the efficient management of construction operations. On the contrary, late detection of onsite operations can be problematic, leaving insufficient time for members of the project team to react which can result in negative impacts on project cost and schedule [1]. Real-time information can provide feedback to support tracking project operations [1-5], safety management [5], productivity analysis, and progress reporting [1,6]. This is especially critical for tracking activities with highly dynamic nature such as lifting activities [7]. 
Using the conventional manual progress reporting methods is time-consuming, labor-intensive and may not be accurate, even for small projects. Besides, the integration of manual data collection and available electronic interfaces can be a challenging task [1]. To overcome the drawbacks stated above, one can make use of automated site data acquisition for schedule updating and progress reporting. Such automation can provide project management teams with needed timely information on the status of onsite construction operations with sufficient accuracy [8]. The use of various remote sensing (RS) technologies for onsite automated data-capturing can result in more robust progress reporting. The captured data can be compared to related as-planned data to perform progress reporting [6,9].

This article encompasses four main areas: an overview of research on automated site data acquisition, standalone RS technologies, integrated use of RS technologies and a general discussion. It is based on a carefully selected set of articles from 20 diverse scientific journals and a couple of conference proceedings in the domain of construction engineering and management. In this search, keywords such as 'remote sensing', 'automated data acquisition', and 'progress tracking' were used. A total of 56 selected studies are used in this review, among which over $50 \%$ were published within last five years. Table 1 presents a list of selected journals and conference proceedings.

Table 1. Source journals, conferences, and the publications used in this review

\begin{tabular}{cc}
\hline Journal/Conference & Quantity \\
\hline Automation in Construction & 8 \\
Advanced Engineering Informatics & 4 \\
Journal of Computing in Civil Engineering & 2 \\
Applied Sciences & 2 \\
Advances in Informatics and Computing in Civil and Construction Engineering & 1 \\
Computing in Civil Engineering & 1 \\
Construction Management and Economics & 1 \\
EURASIP Journal on Embedded Systems & 1 \\
Journal of Construction Engineering and Management & 1 \\
Journal of Construction Engineering and Project Management & 1 \\
Journal of Information Technology in Construction (ITcon) & 1 \\
Journal of IET (The Institution of Engineering and Technology) & 1 \\
Measurement & 1 \\
Remote Sensing & 1 \\
Robotica & 1 \\
Sensors & 1 \\
Visualization in Engineering & 1 \\
IEEE Transactions on Computers & 1 \\
IEEE Transactions on instrumentation and Measurement & 1 \\
IEEE Transactions on Intelligent Transportation Systems & 1 \\
Conference and congress (ISARC, CSCE, ICRA, etc.) & 12 \\
Thesis & 6 \\
Online archives & 6 \\
Total & 56 \\
\hline
\end{tabular}

\section{Overview of Research on Automated Site Data Acquisition}

Most previous attempts in this field were focused on single-sensor models which are not usually applicable for the entire duration of a project $[10,11]$. The literature reveals that the use of a single source of sensory data does not provide sufficient information about the status of onsite construction operations. For example, the point cloud data captured by laser scanners require a direct line-of-sight and they will become less effective as the project progresses and the site becomes obstructed due to increased congestion. In this way, the usage of another data acquisition technology could help to alleviate the drawbacks associated with individual usage of each RS technology in each of the project execution phases. That integration provides more timely and accurate information due to the complementarity and possible fusion of the captured data [3]. Further incorporation of captured 
data with building information modeling (BIM) can lead to more robust progress reporting $[8,9]$. Despite current progress, obstacles still exist for achieving a practical and comprehensive automated solution for onsite progress tracking [6].

Indoor tracking of the construction operations has some difficulties associated with a wide range of materials and structural objects that need to be recognized [6]. Activity tracking was another area of interest in recent years. In fact, an automated progress reporting system based on both activity and material (or objects) tracking has some advantages over systems which are solely based on object tracking [8].

In this article, the latest efforts on automated data acquisition are reviewed. More specifically, methods that integrate various RS technologies are investigated. For instance, the integration of the radio frequency identification (RFID) technology with GPS-based technologies are reviewed to enhance outdoor tracking of resources [12,13]. Data fusion of imaging technologies (e.g., laser scanning and photogrammetry) with technologies such as RFID, UWB, and GPS have also been investigated recently $[14,15]$.

\section{Standalone Remote Sensing (RS) Technologies}

There are a wide range of RS technologies for data acquisition on site. Each of these technologies has its capabilities and drawbacks based on the domain of applications. Selection of one particular technology can vary depending on the type of information required, the accuracy and the environment in which the data will be acquired [16]. One group of these RS technologies includes real-time location systems (RTLSs) such as GPS, robotic total station (RTS), and ultra-wideband (UWB). These technologies generally function based on the time-of-arrival (TOA) principle, where the propagation speed and the propagation time of a signal directly lead to a corresponding distance [10]. Recently, there are some efforts to localize and track people and objects using Wi-Fi-/Bluetooth/BLE technologies for the purpose of occupancy measurement. In fact, by installing some receivers in a target area, the location and number of detected items are estimated using held and/or worn smart phones, Bluetooth headsets, and smart watches. It is done by tracking the changes in the received electromagnetic wave patterns and compare them with calibrated models to count the number of workers or vehicles on site, and to provide rough information about their location and behavioral algorithms. However, due to some legal restrictions for using smart phones on site and not fully accurate results of this technology its use in construction industry is not common yet [17-19]. In a different context, barcode and radio frequency identification devices (RFID) proven to be useful technologies for material identification with applications in tracking resources for a supply chain management system [20,21]. Vision-based technologies such as photo/videogrammetry and laser scanning have also found applications in construction industry for making 3D as-built models of site which provide sufficient information for an automated progress reporting [1]. Table 2 provides a brief overview of these technologies along with their capabilities, limitations, and accuracy. 
Table 2. Capabilities and limitations of standalone remote sensing (RS) technologies

\begin{tabular}{|c|c|c|c|c|}
\hline RS Technology & Capabilities & Limitations & Accuracy & Refs. \\
\hline $\begin{array}{l}\text { GPS, RTKGPS }{ }^{1} \\
\text { and GNSS }\end{array}$ & $\begin{array}{l}\text { (1) Global access in an outdoor environment } \\
\text { (2) RTK GPS provides centimeter-level } \\
\text { positioning accuracy in real time over } \\
\text { long distances } \\
\text { (3) Flexibly and reacting quickly based on the } \\
\text { needs in a construction site }\end{array}$ & $\begin{array}{l}\text { (1) The acquired data are limited to position and time of } \\
\text { objects, and not the identity or type of activities } \\
\text { (2) Delays in data processing and transferring (an important } \\
\text { issue specially for real-time operations such as equipment } \\
\text { tracking or kinematic surveying) } \\
\text { (3) Multipath errors in the congested environment } \\
\text { (4) Signal blockage and building obstructiveness in an } \\
\text { indoor environment }\end{array}$ & $\begin{array}{l}\text { Almost one meter } \\
\text { for GPS and } \\
\text { few centimeters } \\
\text { for RTKGPS }\end{array}$ & {$[5,22,23]$} \\
\hline $\begin{array}{l}\text { Robotic total } \\
\text { station (RTS) }\end{array}$ & $\begin{array}{l}\text { (1) Enhanced tracking and automation } \\
\text { capabilities in the positioning of objects } \\
\text { (2) High positioning update rate } \\
\text { (3) Higher vertical positioning (elevation) } \\
\text { accuracy than unaided GNSS and } \\
\text { GPS-based technology }\end{array}$ & $\begin{array}{l}\text { (1) The high investment } \\
\text { (2) Limited capability to track only one point at a } \\
\text { particular time } \\
\text { (3) Positioning errors will occur if the angles and distances are } \\
\text { not captured simultaneously }\end{array}$ & $\begin{array}{l}\text { Down to few } \\
\text { millimeters and } \\
\text { arc seconds }\end{array}$ & {$[7,24]$} \\
\hline Barcode & $\begin{array}{l}\text { (1) Reasonable price } \\
\text { (2) Straight forward usage with standard } \\
\text { implementation protocols } \\
\text { (3) Speeding up the computer data entry } \\
\text { (4) Portability }\end{array}$ & $\begin{array}{l}\text { (1) Very limited reading range } \\
\text { (2) Sensitivity to the harsh environment }{ }^{3} \text { (e.g., tags are not } \\
\text { readable if covered by snow) } \\
\text { (3) Low data storage capacity }\end{array}$ & N.A & {$[20,21,25]$} \\
\hline RFID & $\begin{array}{l}\text { (1) Longer range than barcodes (up to } 100 \mathrm{~m} \text { for } \\
\text { Ultra-high frequencies) } \\
\text { (2) Non-line-of-sight } \\
\text { (3) Providing cost efficient location information } \\
\text { (4) Tags are light and easy to attach } \\
\text { (5) Unlike barcodes, RFID tags are durable in } \\
\text { construction environments } \\
\text { (6) Batch readability of tags making } \\
\text { identification process much more efficient }\end{array}$ & $\begin{array}{l}\text { (1) Lack of accuracy and difficulties for 3D positioning } \\
\text { (2) Calibration difficulties (e.g., the need for a path loss model) } \\
\text { (3) Affected by multipath effect } \\
\text { (4) Problems associated with simultaneous identification of } \\
\text { many tags } \\
\text { (5) Active tags are fairly expensive. } \\
\text { (6) Plus, relatively wide range may cause obstruction } \\
\text { object detection } \\
\text { (7) The need for battery replacement in active tags } \\
\text { (8) Influenced by metal and high humidity specially in } \\
\text { high frequencies }\end{array}$ & $\begin{array}{l}\text { Down to few } \\
\text { meters for } 2 \mathrm{D} \\
\text { localization }\end{array}$ & {$[4,5,11-13,21,25-30]$} \\
\hline
\end{tabular}


Table 2. Cont

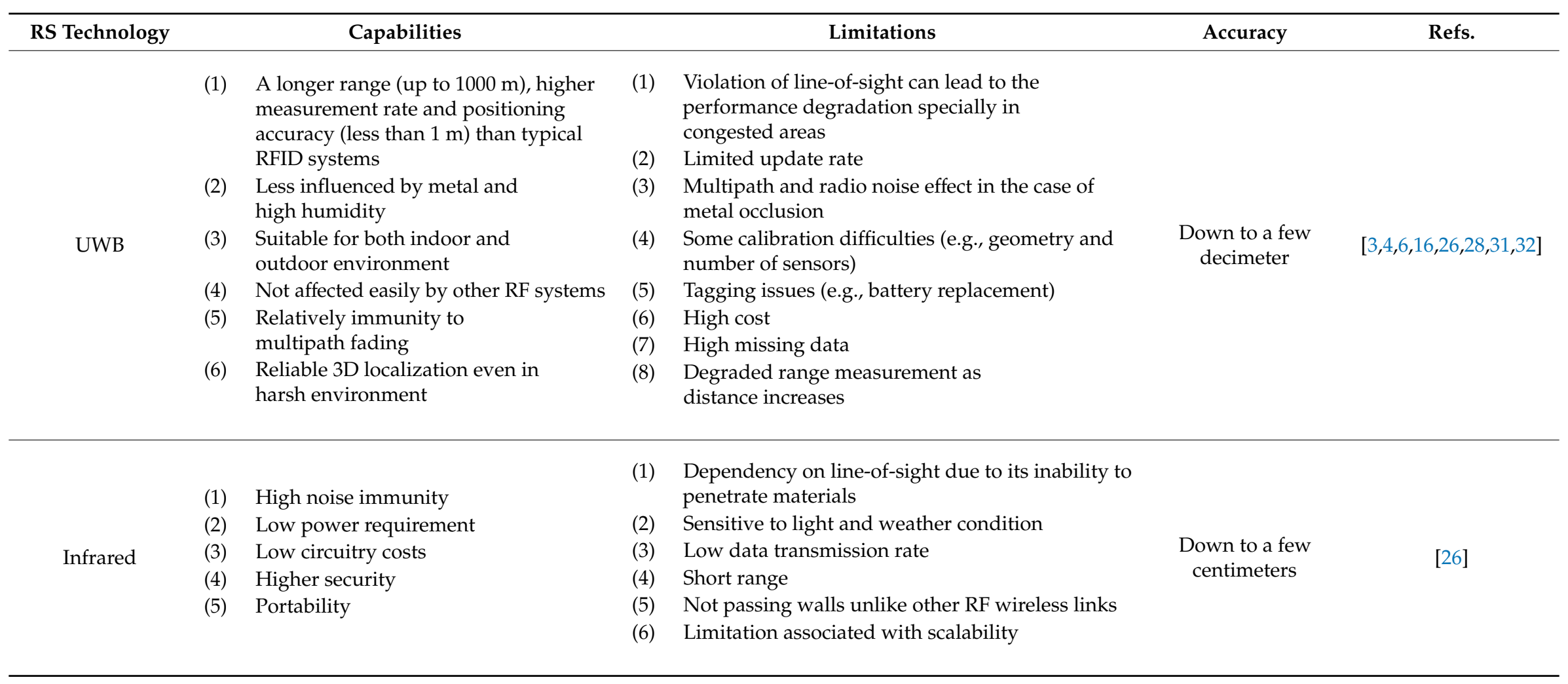


Table 2. Cont

\begin{tabular}{|c|c|c|c|c|c|c|}
\hline RS Technology & & Capabilities & & Limitations & Accuracy & Refs. \\
\hline Photo/Videogrammetry & $\begin{array}{l}(1) \\
(2) \\
(3) \\
(4) \\
\\
(5) \\
(6) \\
(7)\end{array}$ & $\begin{array}{l}\text { Straightforward configuration } \\
\text { Cost-effective field data collection } \\
\text { Portability } \\
\text { Provides information about the } \\
\text { material, texture, and color of the } \\
\text { target object } \\
\text { High update rate } \\
\text { Well known internal geometry } \\
\text { Good interpretability }\end{array}$ & $\begin{array}{l}\text { (4) } \\
(5) \\
(6) \\
(7)\end{array}$ & $\begin{array}{l}\text { Calibration difficulties (e.g., sensitive to the } \\
\text { surrounding light condition, visual occlusions, } \\
\text { and moving background) } \\
\text { Mirror effect caused by reflective surfaces } \\
\text { Less accurate than laser scanners in generating } \\
\text { point clouds } \\
\text { Problems associated with computing depth in } \\
\text { 3D modeling } \\
\text { Difficulty in identification of objects with } \\
\text { unclear geometric configurations } \\
\text { Computational complexity of } \\
\text { photogrammetric surveying } \\
\text { The need for another technology (e.g., } \\
\text { RTKGPS or RTS) to provide the geo-referenced } \\
\text { inputs for photo-based 3D modeling }\end{array}$ & $\begin{array}{l}\text { Around } 1 \% \text { error } \\
\text { in volumetric } \\
\text { measurement }\end{array}$ & {$[1,5,7,25,28,33-35]$} \\
\hline
\end{tabular}

(1) High accuracy in generating point clouds

(2) Simple and well-defined internal coordinate system

(3) Homogeneous spatial distribution of range points

Laser scanning

(4) Ability to scan actively in darkness and shaded areas

(5) Ability to measure areas without texture

(6) Ability to scan a large area
(1) High cost of laser scanners

(2) Scanning is a time consuming process

(3) Occlusion problem and the need for a clear line-of-sight

(4) More post-processing effort is required if the Around $2 \%$ error device moves in volumetric

(5) Limitations associated with modeling of edges measurement and [28,34,36-39] and linear features

(6) High storage capacity is needed in ranging

(7) Not suitable for modeling moving objects measurement

(8) Not providing information about type, texture, and color of the scanned objects

(9) Eye-safety distance concerns

${ }^{1}$ Real-time kinematic GPS, ${ }^{2}$ global navigation satellite system, ${ }^{3}$ extreme cold or hot weather in an outdoor environment. 


\subsection{GNSS-Based Technologies}

The RS technologies which operate based on signals received from satellites have various applications in civil engineering at global, regional, and local levels. Here we focus on the local applications of satellite systems in construction engineering and management, with emphasis on the global positioning system (GPS) as a real-time location tool. The most recent GPS-based systems with applications in outdoor positioning and tracking of resources are global navigation satellite system (GNSS), real-time kinematic (RTK), GNSS, and Russian GLONASS technology. These systems benefit from widespread access to the free global satellite system. However, since an unobstructed line-of-sight to the air is needed, the application of these systems for indoor tracking of resources is challenging in confined areas. Plus, relatively high cost of the accurate GPS receivers is another limitation of these systems for usage in a large-scale level. Below is a brief description of these tracking technologies along with their limitations and capabilities.

\subsubsection{GNSS and Differential GNSS Techniques}

The GNSS is part of a satellite application, providing signals from space that transmit positioning and timing data to GNSS receivers installed on the construction equipment. These receivers then use this data to determine the equipment location [5]. A typical 3D GNSS system, employed in a construction equipment consists of a roving receiver and a reference station [23]. The receiver located on the body of a construction vehicle receives signals from GNSS satellites to determine its position and direction. Besides, in a differential GNSS system the use of a reference GNSS station can enhance the location accuracy by sending corrections to the construction vehicle using a radio modem. These corrections can also be sent to a central unit over the internet through a GPRS modem connected to the levelling system. Furthermore, in a 3D GNSS levelling system, some highly resistant sensors are stuck on the body of a vehicle which make it possible to control any specific action demanded from the equipment in real-time. For applications related to the road pavement which need high accuracy in surface leveling, a 3D control unit processes the signals received from GNSS satellites and the reference station compares them with the stored designed values for quality measurement. A reference station is able to control any number of construction vehicles via onsite radio connections. The accuracy of such a system is around $2-3 \mathrm{~cm}[23]$.

\subsubsection{RTK GNSS}

One of the most recent innovations in using the GPS signals is the RTK GPS or RTK GNSS. It is a technology that provides position accuracy close to that achievable with conventional carrier-phase positioning, but in real-time. The RTK GPS employs a method of carrier-phase differential GPS positioning with centimeter-level positioning accuracy. It consists of a reference station and a roving station with a single or dual frequency GPS receiver. The GPS reference station sends carrier-phase and pseudorange data over a radio link to the roving station. Both single and dual frequency GPS receivers can be used, however, dual-frequency systems usually have faster ambiguity resolution and higher positioning accuracy over longer distances. To achieve the best results, the reference station antenna should be mounted in a location with less barrier and the radio link antennas should be as high as possible in order to maximize the link coverage [22].

Some RTK installations use combined GPS receivers at the reference and roving stations. Besides, a faster ambiguity resolution and higher positioning accuracies can be achieved by using GLONASS data in addition to GPS. However, the data collected at the reference station reaches the rover after some delays which causes some troubles for a real-time operation. This is mostly because of the need for the data to be formatted, packetized, transmitted over the link, decoded, and passed on to the roving receiver's software. This cannot all occur simultaneously and so there are some delays (latency) which depending on the link data rate might be up to two seconds. This delay might be acceptable for some static-point surveying applications, but it is not appropriate for kinematic 
surveys or for equipment navigation. To address this issue, the rover can extrapolate reference station measurements based on its own current measurements with an appropriate filter using an intelligent algorithm. This method can reduce solution latency to less than a quarter of a second, but accuracies are still limited to a few centimeters [22].

\subsection{Robotic Total Station (RTS)}

A RTS system adds tracking and automation capabilities to enhance localization and surveying applications on site. It consists of an electronic transit theodolite which is integrated with an electronic distance measurement (EDM) unit in order to measure both slope distance and angle from a prism attached to a target object. An on-board computer does the tasks associated with data collection and calculations pertinent to triangulation. The distance is measured by counting the integer number of wavelengths in a modulated infrared carrier signal, generated by a small solid-state emitter within the instrument's optical path. The angle is measured by electro-optical scanning of extremely precise digital barcodes etched on the rotating glass discs within the instrument. These localization devices have the angular accuracy of around one arc second, and distance measurement accuracy of one millimeter in static mode and few millimeters in dynamic mode with a high positioning update rate around 20 times per second. In fact, these systems are available in both statistic and dynamic mode for tracking and measurement of a steady and moving object respectively. For the latter one, positioning errors will occur if the angles and distances are not captured simultaneously depending on the vector and velocity of the moving target. To overcome this inherent error, manufacturers have developed total stations in which the angle and distance information are synchronized or they are with low latency [24].

Comparing RTS with technologies such as RTK GPS that we discussed earlier in this chapter, RTK GPS offers an alternative technology to RTS, but its reliability and accuracy in positioning a specific moving object are questionable. Furthermore, the RTS is one of the most accurate on-site survey instruments in the market with accuracy in millimeter level [7]. High vertical (elevation) accuracy is another factor that makes this system a better option than an un-aided GNSS system. These make RTS a very good choice for fine and finish grade applications. Moreover, a total station does not require line-of-sight to the satellites. It can be replaced with GNSS technology in case of obstructions or doing underground operations. Manufacturers have made it easy to switch between GPS and total station sensors for added flexibility. Having said that, total stations are constrained by the required line-of-sight between the total station device and the target prism which is installed on the machine or other resources. Moreover, their limited capability to track only one point at a particular time is another challenging issue [7]. Besides, their limited range can also become an issue. Some manufacturers have overcome this constraint by stringing total stations together in order to control an operation over greater distances without any interruption [24]. High investment required for implementing the devices of this type with acceptable performance specifications is another disadvantage of using these devices for localization purposes.

\subsection{Barcode}

Barcode is one of the most used technologies in industry for data entry with much less error rate and higher speed than those fully dependent on a human operator. A system using barcode technology consists of four main modules including barcode labels, scanner, decoder, and a database for gathering and analyzing information [20]. The barcode labels are available in one-and two-dimensional formats. Generally, the capacity of two-dimensional barcode systems is about 100 times larger than one dimensional system. However, one-dimensional barcodes are more common in industry by having a more accepted governing standard. The scanners are usually available in three main categories such as wand scanners, charged couple devices (CCD) scanners and laser scanners. They are categorized based on maximum length of barcode to be read, maximum distance away from barcode label, ability to read barcode labels attached to irregular objects and scanning reliability. Although wand scanners can read longer barcode labels, laser ones are considered superior with regard to all other criteria 
mentioned. Accordingly, laser scanners were used as data collection devices in the developed system. Decoders, on the other hand, receive the digitized barcode signals from scanners and then decode them to the correct data. Decoders are available in both fixed and portable versions; however, the latter one is more common in construction projects because of the distributed locations of activities in a construction project. Finally, the data are transmitted to the hub computer over a wired or wireless network [20,21].

A practical application of a barcode system in the construction industry is documentation of engineering data. In this way, barcode labels are produced and, then get assigned to each document. After that, coded information is scanned and transmitted to the database. For retrieving the information, developed queries are utilized and results will be shared with industry practitioners. Besides, the performance of this system can be improved by using feedbacks from industry practitioners. A system which utilize this technology is expected to improve productivity and to save time and cost of various tasks such as procurement and document control $[20,25]$.

\subsection{RFID}

A typical RFID system consists of two components: readers and tags which are operating at a specific frequency, ranging from low frequencies to super high frequencies (e.g., from $125 \mathrm{KHz}$ to over 5.6 GHz). RFID tags contain a microchip and an internal antenna with a specific ID and other data associated with an item, to send them to a reader upon its request. Based on their power source, tags can be distinguished as passive, active, and hybrid. Passive tags need to be activated by the electromagnetic energy that a reader emits. Therefore, they have shorter reading ranges and smaller data storage capacities. Active tags, on the other hand, rely on internal batteries for power supply, which increases their reading ranges significantly and enables additional on-board memory and local sensing and processing capacities. Hybrid tags also need to be turned on by a signal such as a satellite wave to transmit the data $[1,11]$. However, the cost of active tags is higher than the cost of passive tags and a battery replacement plan is also required [21]. Tags also can be categorized into two groups: read-only tags, on which data cannot be updated and, read/write tags with a memory that can be updated with new data. RFID readers, on the other hand, consist of a transceiver and an antenna which read data from tags (also write data on tags), and transfers the collected data to a host computer or server for future retrieval and analysis. The readers are available in both statistic format and hand-held roving devices based on the application required on site. With an antenna, the communication between the transponder and the transceiver is possible; however, its shape and dimension influence its performance characteristics such as frequency range. Available frequencies include low frequency (LF), high frequency (HF), and ultra-high frequency (UHF). Super-high frequency (SHF) or microwave is also used in some cases. Presently, UHF is the most widely used, because UHF passive tags offer simple and inexpensive solutions and most active tags operate on UHF [11].

Many researchers have investigated RFID technology with great capability in automatic identification and tracking of tagged objects on site. Moreover, it is applicable for the built environment due to its non-line-of-sight capability, wireless communication, and on-board data storage capacities [4,11]. Besides identification capability for tracking purposes on site, an RFID system can also be used for 2D localization of tagged objects. Studies have investigated the application of the RFID system for 3D localization of materials on site, however, only using RFID sensors faced some difficulties since a high density of reference tags in elevation are needed [30]. There are three main methods to localize an object using RFID sensors including triangulation, proximity, and scene analysis $[4,26]$. Triangulation determines the position of an object by measuring its distance from several reference positions (e.g., known position of some reference tags). Then, by using techniques such as trilateration, the position of other tags can be determined. The proximity method includes the measurement of the nearness of a set of neighboring reference points with fixed and known locations which are close to the target. In fact, in this method, the distance of the target object from reference points is not actually measured, but its presence within a certain range is only determined. Therefore, it has 
a rough localization accuracy. The scene analysis method estimates the location of a source of the signal (target object) by using a pre-observed data set of the monitoring scene with defined features in an electromagnetic signal characteristics map. Having said that, employment of this technique is not practical for construction sites with dynamic environment since it requires extra information and data storage [4]. Many researchers have used RFID for automated data acquisition from the site. For instance, in [4] a two-step process for localization of objects using a hand-held RFID reader and passive tags was used. In fact, they employed some reference tags for localization of RFID reader and then the location of the tags determined by using a path loss regression model and trilateration technique. Unlike barcodes, RFID tags can withstand harsh conditions with a much longer reading range which facilitates the automated material tracking on site. Having said that, its performance is degraded in vicinity of metal and liquid at high frequencies (e.g., UHF and microwave) [21].

\section{5. $U W B$}

Ultra-wideband (UWB) technology is a real-time location system (RTLS) which was initially developed for military use in the 1960s [16]. Its performance is almost similar to the active RFID system; however, it uses very narrow pulses of radio frequency (RF) energy which are occupied in a wide bandwidth for communication between tags and receivers. That is especially suitable for environments in which the multipath effect may happen. Like RFID technology, here we have some tags which are attached to the target objects, some receivers to read the tags data and a central hub with software to receive and analyze all these data for localization. However, the decision on choosing an appropriate type of tags really depends on the asset type (e.g., equipment, material, or people), its velocity and its operational environment. Various types of UWB sensors use different positioning techniques to estimate the tag's position. These techniques include the time of arrival (TOA) or time of flight, angle of arrival (AOA), time difference of arrival (TDOA), and received signal strength (RSS). AOA has some advantages over TDOA as it does not require synchronization of the sensors nor an accurate timing reference. While TDOA is less sensitive to changes in setup calibration but it still requires more cabling to have an accurate timing reference [3]. TOA, on the other hand, is more accurate when the two devices are in a clear line-of-sight, however, RSS is less influenced by changes in placement of objects in the environment.

A UWB system has two operational modes for data communication including wired and wireless modes. The wired mode in which all sensors are connected with timing cables can localize the tags using both AOA and TDOA techniques. While in the wireless mode, TOF and AOA are usually used for localization. It has been investigated that removing the timing cables will decrease the accuracy, but still a location accuracy of less than one meter is provided by the UWB system. However, in terms of spatial disruptions on the monitored area, the wireless mode is preferred since it imposes minimal spatial disruption due to less required cabling [16]. A number of companies [40-42] have provided newer versions of these sensors with better accuracy and reliability for various applications in construction. For example, Trek1000 Evaluation Kit is a product of an Irish company [42], which utilizes an atomic timer embedded in their PCB board that provides a high positioning accuracy in the range of a few decimeter for these wireless sensors. The product that is manufactured by this company is almost eight times less expensive than the available commercialized sensors, however, it is still not a final product with a protected enclosure. In [33], a set of experiments showed better performance of this product over other sensors by a mean ranging error of around $12 \mathrm{~cm}$ for this type of sensors. Plus, removal of the outlier data and using some data filtration techniques can improve both ranging and localization accuracy. Figure 1 illustrates a schematic view of these UWB sensors along with other RS technologies for automated onsite data acquisition.

Several researchers have investigated the possibility of using this technology for identification, localization, and tracking of construction operations. They focused mostly on evaluating real-time tracking of workers, equipment, and materials in indoor and outdoor environments [3,16], and further on improving the quality of data received by the UWB system. It was shown in [16] that placement 
of static reference tags in the field can improve the positioning measurement of UWB sensors. Furthermore, the geometric configuration of the reference point and the receivers could improve the accuracy of the range measurements. Applying a suitable data enhancement method such as Kalman filter (KF) can also improve the localization accuracy. In fact, it mitigates the data uncertainties through prediction and update steps which would result in an optimal estimation of the desired parameter by using probability density functions and noise factors. In [36], the authors employed a particle filter to improve the localization accuracy of three different UWB devices by using a Bayesian filtering approach. In their experiment, they used the parameters in line-of-sight (LOS) condition to improve estimated locations in non-line-of-sight (NLOS) condition by using a combined Gaussian and Gamma distribution. In [43], the integration of extended Kalman filter (EKF) and extended unbiased finite impulse response (EFIR) filter was used in order to have more optimized and robust results. The integration of these two filters provided both robustness and accuracy required for localization with UWB sensors in a noisy environment like a construction site. The fusion of these two filters was based on defining a time-varying weighting factor for each of them. In fact, before reaching a specific time in the EFIR, the EKF was the active filter to find the optimized solution. Subsequently, the integration of these two filters comes to play. Therefore, the roving tag position is calculated by the summation of these two weighted factors in each estimated position. Researchers in reference [3] also showed that by attaching more than one tag to each object, and then averaging the positioning data acquired by these tags, the localization accuracy will be improved in comparison with the use of one tag. A higher number of receivers on site also increases the accuracy of this system [16].

The UWB sensors are less influenced by metals and high humidity (a limitation of ordinary RFID systems) and require only a few sensors. Comparing with other technologies such as RFID or ultrasound, UWB has unique advantages including longer range, higher measurement rate, better measurement accuracy, and relative immunity to multipath fading $[11,16]$. Besides, UWB offers a wide spectrum and fine time resolution of the signal which results in a more reliable positioning in a harsh environment (e.g., good performance in vicinity of moisture or snow). However, violation of the line-of-sight can lead to a decrement in the signal strength, so an accurate design and sensor placement strategy is needed. Unfortunately, the cost of UWB sensors is still high in comparison with other sensors [31].

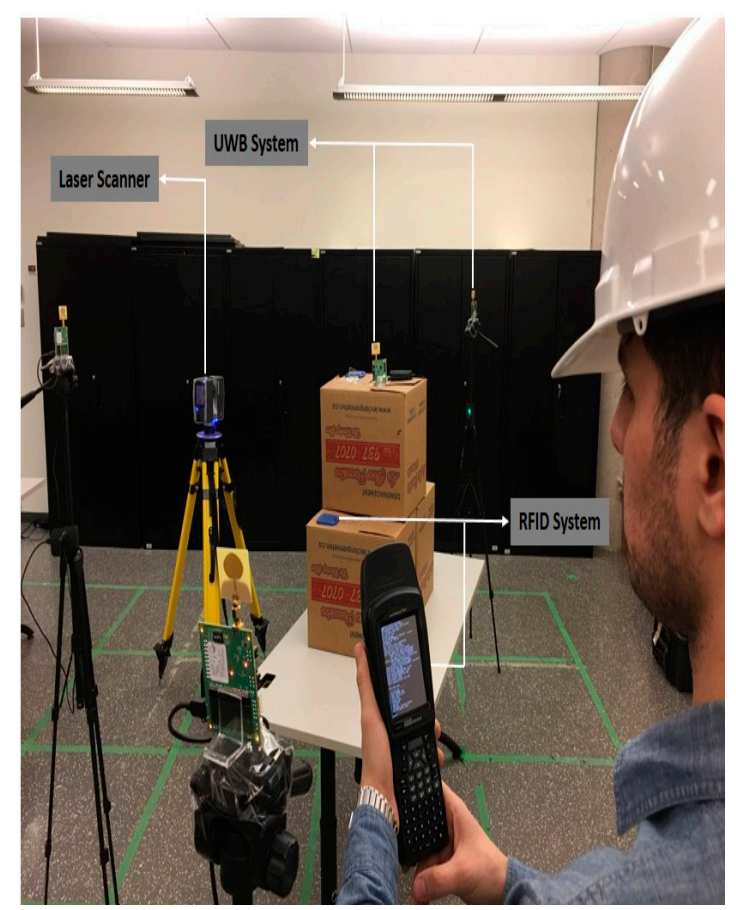

(a)

Figure 1. Cont. 


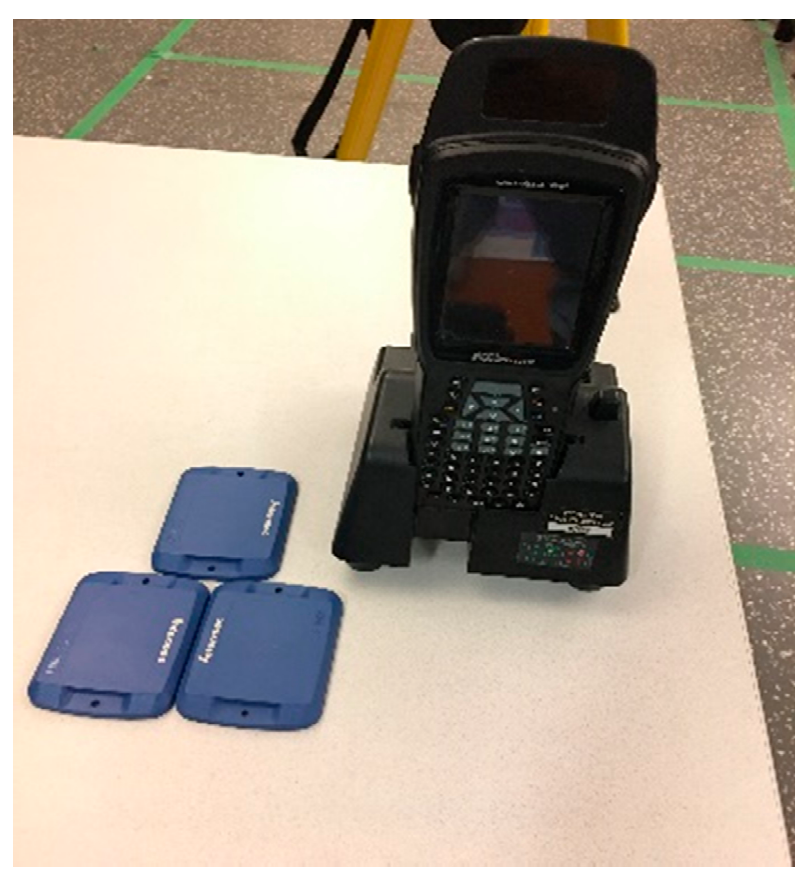

(b)
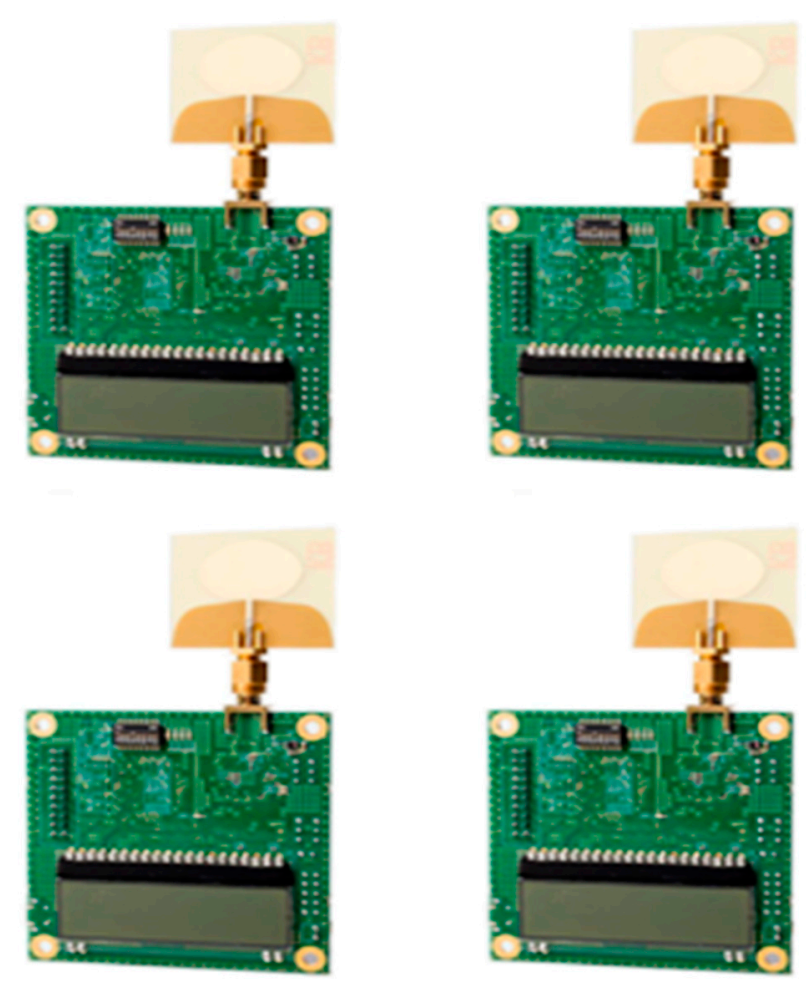

(c)

Figure 1. Cont. 


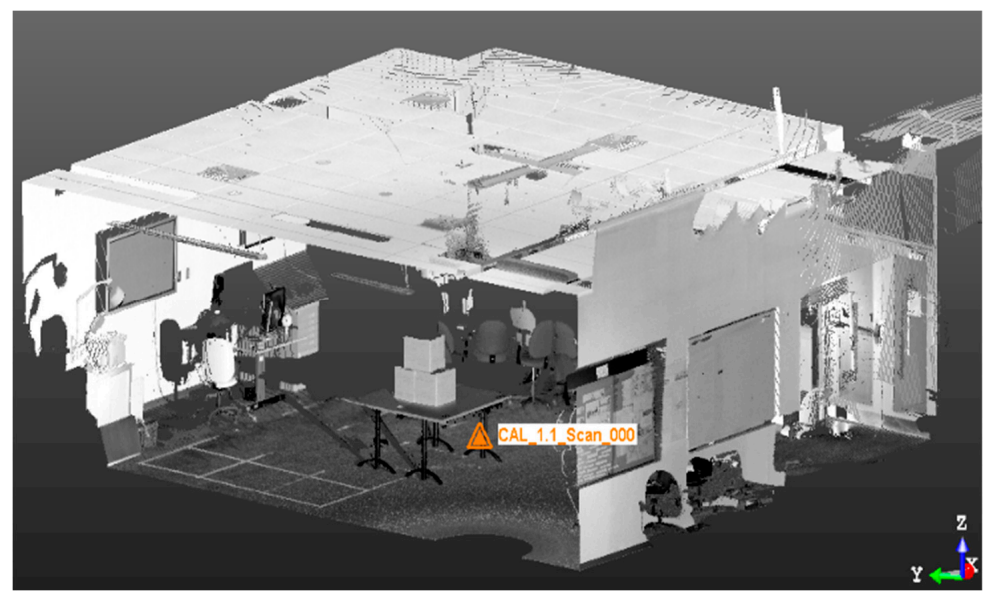

(d)

Figure 1. Various remote sensing technologies. (a) Automated data acquisition on site; (b) RFID system; (c) UWB system; (d) point cloud data for 3D modeling.

\subsection{Bluetooth, Infrared, and Ultrasound}

Bluetooth technology has recently become more popular with the introduction of Bluetooth low energy (BLE) technology in 2010. In contrast to Wi-Fi, the battery usage on the client side is very low for BLE technology which can enable it to work up to three to four years with only a single small battery. However, the fluctuation in the strength of the received signal is a challenging issue when using these sensors. Saying that, due to the inherent nature of the system, it is highly recommended for accurate positioning. Besides, it is incorporating well with other techniques such as filtering techniques and motion sensors [31]. These technologies can also be used to prepare a congestion map which shows the areas onsite with more accident risk or activity conflicts, by information acquired from workers and vehicles behavioral patterns [19].

Infrared is electromagnetic radiation at a wavelength longer than red light. Infrared wireless technology can be used for short and medium range communications and control purposes, however, unlike radio-frequency (RF) wireless links, it cannot pass walls. Ultrasound, on the other hand, is usually implemented by using special microphones in a room. In fact, items that need to be tracked have a tag ID with a speaker attached. While the sound is stopped by the door, wall, or window but it is not stopped by other physical objects in the room such as people. These sensory systems need hardware infrastructure for signal communication and they have scalability limitations. Actually, they are rather focused on high accuracy than ease of deployment. Having said that, it requires a line-of-sight due to its limitation to penetrate in materials [31].

\subsection{Vision-Based (Imaging) Technologies}

Many pieces of research on automated progress reporting have focused on creating 3D models or point cloud models for both indoor and outdoor environments. These point cloud data are generated by using image frames or laser scanning data of the site. Generally, laser scanners can provide more accurate point cloud data but with more processing effort [1]. However, in recent years due to the availability of high-resolution cameras, more powerful computers, and new advancements in image processing techniques more accurate and real-time point clouds are generated by only using image frames.

\subsubsection{Photo/Videogrammetry}

Photogrammetric surveying has been widely used in construction engineering and manufacturing for 3D modeling, localization, and map production. Virtual reality (VR) and augmented reality (AR) research have referred photos as the most straightforward and cost-effective inputs for field 
data collection in construction management. The photogrammetric technique advantages over other traditional surveying methods include: (1) non-contact measurements, (2) relatively short turnaround cycle of data processing and modeling, and (3) measuring multiple points at one time [7]. Furthermore, researchers have extended photogrammetry into videogrammetry. Since video frames are sequential, pixels available in each frame are progressively reconstructed based on the previous frame(s). This characteristic of videogrammetry has made it possible to reconstruct various elements more quickly in civil infrastructures [1]. However, an extra video post-processing effort is necessary to match the time-stamped videos of each camera together. Generally, photogrammetry includes putting some cameras with a standard distance and angle of view from each other in a jobsite. Saying that, by advancement in simultaneous localization and Mapping (SLAM) techniques, the point clouds can also be generated by a moving camera. For registration of photo frames together, there is a need to have some stationary site control points (usually more than three) in each photo frame for 3D modeling. So, images taken from different angles are used to build the 3D model, by pairing and matching some common points in the two or more neighbor frames. It should be noted that the time differences between the photos and the control point survey data must not exceed the preset value (e.g., one second), otherwise, the photos and the survey data are discarded for ensuing 3D modeling. The 3D model coordinate system is set up by selecting an arbitrary origin and scale. The image and object coordinates are then evaluated based on defining specific parameters in related photogrammetry equations. The scale of the model is determined by using coordinates of some points which are determined by a terrestrial station such as robotic total station (RTS) unit. Reducing the modeling time and cost by generating the point cloud from both photos and structure from motion (SfM) method is also an acceptable approach. In fact, the point cloud-based application is aimed to reduce the effort in producing $3 \mathrm{D}$ as-built models. However, the model object must be stationary during the laser scanning process while removing the noise data (redundant or irrelevant information) requires considerable time and expertise. Thus, point cloud-based technologies are not suitable for modeling a particular moving object on a near real-time basis on site. In order to solve some of the limitations mentioned above, developing an alternative system, which can directly track and survey target points by use of synchronized RTS units would be useful. Such a system can automatically provide the geo-referenced inputs needed for photo-based 3D modeling [7]. This platform is reviewed in the section for integrated photogrammetry with RTS technologies.

Recent studies on this field have been focused on videogrammetry to create point cloud data through SLAM technique. In [35], a platform was designed for a real-time progress reporting in which a client connects to Wi-Fi and takes a video record of the site. Then this video (or image frames) is sent for mapping of the construction site by producing the site point cloud data. Then, through the reconstruction step, a dense point cloud with enough details is generated in which a mapping of the visual objects available on site is achieved. Then, for any new query image, the location/camera pose of the input image would be retrieved by using a feature-based matching with the base 3D point cloud map generated earlier. Finally, by aligning the parameters of the 3D point cloud with a geo-referenced BIM model, global localization of the frames can be estimated.

\subsubsection{Laser Scanning}

Using 3D point clouds produced by laser scanners has already been used as a standard practice for generating as-built information in construction, rehabilitation and facilities maintenance in areas ranging from process plants to historical preservation and structural health monitoring [9]. These devices use near-infrared $(0.7-0.9 \mu \mathrm{m})$ wavelength in order to create 3D point clouds of the objects available in an environment. There are some important factors which determine a laser scanner performance such as angular resolution, scanning speed and the range required [37]. Clearly, the quality of the point clouds usually depends on the number of times doing the measurement for each point. As the number of measurements per point increases, the quality of the point cloud increases. Terrestrial laser scanning (TLS) is one of the best available technologies to capture 3D information on a project with high 
accuracy and speed. It has made it possible for construction managers to track progress, control quality, monitor health, and last but not least to create as-built 3D models of the site. Many studies have been focused on the interface between laser scanned data and a 3D model such as CAD or BIM models. For instance, in [9] a 3D CAD model was used as a prior source of information for detecting objects from the point clouds. However, these approaches are unable to identify objects which are not in their exact locations pre-described by as-designed model or located in the distance greater than a tolerance value specified in the object recognition software. Furthermore, they are incapable of providing sufficient information about non-structural activities such as the welding, inspection, piping system, etc.; however, BIM systems may help to facilitate these operations in future [6]. In fact, with the advent of 3D BIM, many newer approaches actively use the 3D information contained in BIM models to develop supervised object detection and recognition algorithms that can process the point cloud data more effectively. Nonetheless, the process of recognition, detection, and identification of objects in 3D TLS data remains an open challenge with marketed software while it is needed to be more studied in order to enhance the compatibility of these technologies with available software. The objective of the process of 3D laser scanning is to scan a physical space or site to create an accurate digital representation of it. The scan to BIM is a term that refers to the process of scanning an existing building, area or site and creating a 3D model from this data. In fact, this process is proved to be more exact than conventional inspectional data that are usually accomplished using a measuring apparatus. The scan to BIM process can be divided into two stages. The first stage is the fieldwork where the surveying team goes to the site and collects data using their 3D laser scanners. The second stage is the office work where the raw data is calibrated and could data is used to create a 3D model [9].

\section{Integration of Various RS Technologies}

Due to the diversity of the construction resources, harsh environment, and complex nature of construction projects, the data acquired by each RS technology is missing or erroneous. Furthermore, each RS technology has its capabilities and drawbacks that limit its application. In fact, depending solely on a single technology to acquire data on-site in an accurate and timely manner is unreliable. In this way, the integration of various RS technologies together can improve the data acquisition process. Plus, it can help to overcome the limitations of an individual technology and makes it possible to provide the required information even if one of these data sources is not fully available $[16,25]$. This integration could happen in different scenarios. In a typical scenario, the integration includes the separate employment of various RS technologies in different stages of construction activity. Examples of this type of integration are available in [25], in which integrated barcode-RFID and photogrammetry-laser scanning systems are used for resource tracking and quantity measurement on site. In another scenario, the data acquired by each technology could enhance the data acquired by other technologies which result in having more accurate and robust information. An example of the latter integration approach is the data fusion of various RS technologies together or a multi sensor data fusion (MSDF) approach. In fact, data fusion refers to the combining of information from multiple sources in order to improve the quality of information obtained separately from each source. It is utilized to make inference decisions about the state of a construction project based on the data that are acquired from different sources [16]. Therefore, informed decisions and objective assessments of the activities progress on site are not only based on a single data source. In fact, a number of data sources are combined together in order to provide sufficient details about various activities on site. It should be noted that the level of data fusion is an important factor in order to have a more robust and accurate approach [28]. Recent studies in this field are focused on the integration of various RS technologies in order to benefit from identification, localization, and visualization capability of each of these technologies with applications in tracking objects and activities on site. Table 3 shows some of these integrated systems along with their capabilities over standalone usage of each technology and their drawbacks for implementation on site. 
Table 3. Capabilities and limitations of integrated RS systems

Type

Integrated RS System

Capabilities

Limitations

Refs.

(1) Relatively low cost (mainly goes for GPS receiver); barcode: Label $\sim \$ 0.1$, Reader $\sim \$ 100-500$ and GPS: Receiver \$200 Satellite signal free

GPS + Barcode (2) High level of standardization and reliability

(3) More scalable for projects of varying sizes

(4) Straight forward implementation

(1) Need for free access to space for GPS system which makes it unsuitable for interior environment

(2) Limitation of barcode tags in differentiating between items of the same kind

(3) Not fully automated approach

(1) The need for free access to space for GPS system

Positioning systems integration with other sensory data

GPS + RFID

(1) Easier material identification due to non-line-o sight capability of the RFID tags

(2) Providing both identification and localization data simultaneously

(2) A large number of RFID readers are required which increases the cost; RFID Tag \$1-50 and RFID Reader \$1k-5k

(3) Boundary constraint limitations in

cluttered environments

(1) Stability to be used in various construction operations

(2) Real-time tracking and reporting capabilities

Sensor-aided GPS (3) Not sensitive to the ambient environment

(SA-GPS)

(4) Having both location/action

recognition capabilities

(5) Providing continuous update of the location estimates
(1) Obstacles associated with data fusion, coordination, processing, and reduction of data to produce meaningful conclusions

(2) Requiring relatively more time for post processing

(3) Drift inherent to sensors

(4) Initialization and calibration difficulties
(1) Make it possible for tags to communicate with each other

(2) Facilitating the negotiation of RFID

RFID integration RFID + WSN with other sensory data

$$
\text { readers together }
$$

(3) Increased positioning accuracy

(4) Decreased energy consumption in comparison with an individual WSN ${ }^{1}$ system
(1) Sensor does not provide any power until tag is not in the radio frequency field to communicate with reader

(2) Reading range decreases as the system starts $[26,29,48,49]$ 
Table 3. Cont.

\begin{tabular}{|c|c|c|c|c|c|}
\hline Type & Integrated RS System & & Capabilities & Limitations & Refs. \\
\hline & RFID + LS & $\begin{array}{l}(1) \\
(2)\end{array}$ & $\begin{array}{l}\text { Faster and more robust recognition and } \\
\text { positioning of objects in 3D modeling } \\
\text { Alleviating the difficulties associated with the } \\
\text { laser scanners object recognition specially in a } \\
\text { crowded and full of furniture environment }\end{array}$ & $\begin{array}{l}\text { (1) Poor positioning performance when } \\
\text { the objects are in the corner of a room } \\
\text { (2) Laser scanners are relatively expensive } \\
\text { (TLS could cost more than } \$ 60 \mathrm{k} \text { ) } \\
\text { (3) Need for a facility management } \\
\text { database of the available objects } \\
\text { in advance }\end{array}$ & {$[14,39]$} \\
\hline \multirow[t]{2}{*}{$\begin{array}{l}\text { Integration of the } \\
\text { vision-based } \\
\text { technologies } \\
\text { together and with } \\
\text { other RTLS }\end{array}$} & $\begin{array}{c}\text { Photogrammetry + } \\
\text { laser scanning }\end{array}$ & $\begin{array}{l}(1) \\
(2) \\
(3) \\
(4) \\
(5) \\
(6) \\
(7)\end{array}$ & $\begin{array}{l}\text { Less number and time for scanning are required } \\
\text { Less number of digital photos are required which } \\
\text { results in less computational effort } \\
\text { Better object recognition capabilities by adding } \\
\text { depth information from laser data to the available } \\
\text { digital photo planes } \\
\text { Convenient for modeling objects with unclear } \\
\text { geometrical properties (e.g., excavation activities) } \\
\text { More accuracy in the localization of edge points } \\
\text { Providing more details about material, color, and } \\
\text { texture of the objects which improves the } \\
\text { geometry and visual quality of the 3D modeling } \\
\text { Self-calibrating capabilities for cameras }\end{array}$ & $\begin{array}{l}\text { Expensive laser scanners } \\
\text { Problems associated with calibration } \\
\text { and orientation of data acquired from } \\
\text { two sensory sources, e.g., } \\
\text { geo-referencing issues } \\
\text { Less effective when the site is crowded } \\
\text { or covered by enclosures }\end{array}$ & {$[25,28,33,34,38,50]$} \\
\hline & $\begin{array}{l}\text { Photogrammetry } \\
\text { + RTS (robotic } \\
\text { total station) }\end{array}$ & $\begin{array}{l}(1) \\
(2)\end{array}$ & $\begin{array}{l}\text { Enhanced scaling and 3D modeling capabilities } \\
\text { Better measurement accuracy (down to few } \\
\text { decimeter) rather than using individual } \\
\text { photogrammetry techniques }\end{array}$ & $\begin{array}{l}\text { (1) High cost of RTS devices } \\
\text { (2) Synchronization of digital cameras } \\
\text { with RTS devices } \\
\text { (3) Limited capability for tacking multiple } \\
\text { moving objects }\end{array}$ & {$[7,24]$} \\
\hline
\end{tabular}


Table 3. Cont.

\begin{tabular}{|c|c|c|c|c|c|c|}
\hline Type & Integrated RS System & & Capabilities & & Limitations & Refs. \\
\hline & $\begin{array}{c}\text { Photo/videogrammetry } \\
+ \text { UWB }\end{array}$ & $\begin{array}{l}\text { (2) } \\
\text { (3) }\end{array}$ & $\begin{array}{l}\text { More reliability in positioning in comparison with } \\
\text { individual use of each technology by replacing the } \\
\text { data of one sensor with another in case of any } \\
\text { failure in each sensor } \\
\text { More accurate and timely information are } \\
\text { provided by proving more automation and } \\
\text { decreasing computational effort } \\
\text { in photogrammetry } \\
\text { Portability }\end{array}$ & $\begin{array}{l}\text { (1) } \\
(2) \\
\text { (3) }\end{array}$ & $\begin{array}{l}\text { Calibration difficulties specially for } \\
\text { large scale sites } \\
\text { Error propagation phenomenon due to } \\
\text { the intrinsic inaccuracy in } \\
\text { each technology } \\
\text { Less accuracy in comparison with other } \\
\text { geo-referenced photogrammetry } \\
\text { techniques (e.g., using a RTS or } \\
\text { GNSS system) }\end{array}$ & {$[3,32,51]$} \\
\hline & Laser scanning + UWB & $\begin{array}{l}\text { (1) } \\
\text { (2) } \\
\text { (3) }\end{array}$ & $\begin{array}{l}\text { Overcoming challenges associated with the } \\
\text { presence of multipath, NLOS }{ }^{2} \text { propagations and } \\
\text { low visibility (e.g., occlusion, dust, fog, etc.) } \\
\text { Less accumulated error in comparison with an } \\
\text { individual usage of laser scanning technology } \\
\text { Ease of need for prior knowledge or control inputs }\end{array}$ & $\begin{array}{l}(1) \\
(2)\end{array}$ & $\begin{array}{l}\text { Discrepancy between the accuracy of } \\
\text { the UWB mapping and that of } \\
\text { LiDAR mapping } \\
\text { High implementation cost }\end{array}$ & {$[6,15,28]$} \\
\hline
\end{tabular}

${ }^{1}$ Wireless sensor network, ${ }^{2}$ none line-of-sight. 


\subsection{GPS-Based Systems Integration with Other Sensory Data}

The localization capability of GPS sensors could be integrated with the identification capability of technologies such as barcode and RFID for more accurate tracking of materials on site. Plus, integration of the GPS sensors with other aided sensors could provide more accurate and robust information about the site condition and the resources available in it.

\subsubsection{GPS Integration with Barcode}

It includes integration of the barcode and GPS technology for material identification and localization respectively. In this technique, various items are tagged by barcodes and their real-time locations are sent to a central database through Wi-Fi as scanned by a barcode reader equipped with GPS. For instance, in [52] an automated system for material tracking based on the integrated barcode and GPS technology was developed in which the location of materials is determined with three-meter accuracy. In fact, automated and real-time identification and localization of materials make the retrieval of these elements much easier which results in a better material tracking and management system.

The advantage of this tracking technology over other technologies includes relatively low cost, high level of standardization, and scalability for employment in various projects. Barcode labels have been widely used for many applications in industry with defined protocols to be attached to various materials for many years with relatively low cost (see Table 3). However, the reader should be very close to the barcodes which is not practical on many occasions for tracking items on site. Besides, the barcode system cannot differentiate between the items from the same type which decreases the level of detail provided for the identification process [52].

\subsubsection{GPS Integration with RFID}

Integrating GPS with RFID would provide a reasonable solution to benefit the localization and identification capability of both technologies. There are various scenarios to implement such a system in order to have a better outdoor resource tracking and management. In one scenario, the GPS receiver is attached to a roving RFID reader in order to provide information about the position of the RFID reader. By knowing the real-time location of the roving RFID reader, the 2D location of the tagged items are identified by using trilateration technique. Having said that, this technology is not accurate enough for localization of objects in elevation since GPS-based technologies cannot provide accurate information about height. In [12], a system consists of spatially distributed mobile RFID readers equipped with GPS technology was developed which could detect mobile RFID tags. The readers transfer the data to a central server via a communication network in which the server filters the data using a boundary condition-based algorithm to estimate the continuous and real-time 3D locations of tagged objects. In fact, boundary constraints were introduced to facilitate RFID-based localization applications to overcome the challenge associated with unknown tag-reader distance [12,13]. In another experiment [2], researchers tried to improve this approach by integrating the RFID sensors with a real time kinematic (RTK) GPS device. In their system, the location of the reader was recorded as a boundary point, which was acquired by the RTK GPS receiver roving together with the readers. Then, the tag's location was estimated using bilateral method in which by creating four equal-radius spheres centered at four boundary points (i.e., four readers that form the boundary condition with the target tag) and then checking whether an overlapped common volume exists. Besides, they identified two important factors to improve the accuracy of estimations including: (1) the reading angle between a tag's orientation and a reader's shooting direction, and (2) the geometric configuration of the reader locations in each time step. The geometric configuration of the readers was assessed based on a parameter called Spatial dilution of precision (SDOP) which was formulated to improve the localization accuracy [2].

In another scenario, a separated distribution of RFID readers and GPS receivers are employed to benefit the positioning and identification capability of these two technologies. In [5], a system based on 
the integration of RFID and GPS technology was developed in order to enhance the safety of workers on site by improving blind lifting and loading crane operations. The system architecture includes a GPS receiver and an RFID tag attached to the crane hook, and active RFID tags attached to the safety helmet of the workers. During the crane operations, the location of the workers and crane hooks are thus read by the RFID readers installed on the site and then imported to the BIM environment. In fact, their system obtains and compares the real-time position of tower cranes and construction workers and, if any unauthorized worker is detected in a pre-defined risk zone, an order by central hub would be sent to cut off the crane power. In a case study done for this approach, an accuracy of approximately $\pm 30 \mathrm{~cm}$ was recorded [5].

As stated, accuracy is an important factor when deploying a system for data acquisition in a construction site. Therefore, the integration of RFID with other positioning technologies like GPS-based systems is a solution to have higher accuracy in tracking tagged items. Having said that, GPS solutions present some drawbacks in certain environments especially when it comes to the interior of buildings. In order to avoid the limitations of RFID and GPS-based systems, the combination of ultrasound technologies and RFID can improve the positioning system [27,53].

\subsubsection{SA-GPS}

A sensor-aided GPS (SA-GPS) prototype consists of a microcontroller equipped with GPS module, and a number of sensors such as accelerometer, gyroscope, barometric pressure, strain gauges, etc. This prototype consists of some sensors that are installed on various resources on site and using a gateway to communicate together and with a central server. In this way, it can provide more accurate information about site conditions and working hours of the target resources [26,44]. Several studies have investigated the effectiveness of this system for progress estimation and tracking of various objects and activities. In [26], a system based on SA-GPS was designed for tracking and progress assessment of the earthmoving operations. They concluded that there is a considerable decrease in average absolute percentage error in the project progress estimation in comparison with a system working only based on GPS from $12 \%$ to almost 3\%. In a similar study in [47], a smartphone with sensor technologies-such as GPS, accelerometer, and gyroscope-was put inside construction equipment. Then, the time-stamped data for the equipment position, acceleration and angular velocities were collected by using some commercial data logger applications available on the smart phone device. After a feature extraction process in which some features were defined based on the level of details required for an activity recognition process, a subset of originally extracted features were employed to train the supervised machine learning algorithms (e.g., artificial neural networks (ANN), K-nearest neighbor (K-NN), etc.) for activity classification. Finally, through an activity recognition step the classified actions were defined as real actions in the site and these data got used as input for a simulation model. These estimations can also be used for a more accurate and enhanced earned value analysis (EVA) and productivity assessment $[26,44]$. Furthermore, integration of the GPS with inertial navigation system (consists of an accelerometer, a gyroscope sensor and a magnetic compass) can be used to estimate both the position and velocity of a moving object such as construction equipment. However, both GPS and INS estimations have some limitations and errors. In fact, the GPS error is caused by the time difference between the satellite and a moving device, geometrical difference among satellites and multi-paths of the GPS signal. INS, on the other hand, has good capability for precise location-information in short term but the accumulative error caused by environmental disturbances could be too big if it is used for a long time. Therefore, it would be very difficult to properly handle the noises of INS sensors for the precise localization of a moving object since the motion of the mobile object is nonlinear and the noises are not Gaussian. In this way, data fusion of these two sensory sources by appropriate filtering of the velocity-dependent noises in the INS signals would result in a more accurate and reliable data for the purpose of the outdoor tracking of the equipment [45]. In another study [46], other onboard aided sensors (e.g., inertial sensors) in addition to the GPS sensors were employed to investigate the effect of road slope in positioning and tracking of the equipment. They concluded 
that this approach can improve the accuracy and reliability of the equipment position estimations in comparison with approaches based on planar position estimation algorithms. In fact, depending on the planar models for positioning of the equipment in the sites with terrain condition would be more erroneous as the slope and curvature of the road vary. Therefore, it would be possible to improve the accuracy and reliability of pose estimations by compensating for the error due to the road slope. Finally, the integration of the GPS with other aided sensors provides capabilities with applications in the advanced driver assistance system (ADAS) and autonomous vehicles. This can provide more automation in the construction activities which can enhance the productivity and safety of the activities as a long-term objective in the construction industry [46].

Integrating GPS data with aided sensory data can compensate for the limitations in utilizing the individual GPS information. In fact, it would make it possible to capture the big picture of the construction operations by accurately distinguishing between productive, idle and out of service times during construction activity. Furthermore, the idea for using integrated sensors on a mobile phone device can mitigate the difficulties associated with mounting sensors on equipment body (e.g., attachment and detachment of various sensors for every data collection session), dust in a construction site, data storage issues, and unstructured arrangement of resources which makes calibrating of sensors difficult. Furthermore, it can improve inaccurate and unrealistic simulation models which are usually working by static input data and are built upon historical information during the pre-construction phase [47]. Having said that, the process of fusing the data acquired from various sources is an obstacle for achieving a real-time and automated procedure [26,44].

\subsection{RFID Integration with Other Sensory Data}

Only a few technology sets are available for indoor positioning. The reasons for this poor diffusion include high costs compared to the value achieved and technical limitations such as precision, reliability, and performance of such systems. In this way, some researchers have investigated the integration of a wireless sensor network (WSN) with the RFID. This approach provides new capabilities in order to have a better estimation by facilitating the identification of materials located in the scene and estimating their position at the same time [26,27]. The integration of the RFID technology with other RS technologies can also improve onsite information for having efficient management of construction operations during a project lifecycle [27].

\subsubsection{RFID Integration with a Wireless Sensor Network (WSN)}

In a system based on the integration of the RFID and WSN, smart nodes of sensors are employed to identify objects that their sensed information is needed, while the smart node readers detect tagged objects to recall them at a convenient time [26]. In fact, by integrating a large number of sensor nodes with RFID tags and readers, communication of the RFID tags with each other and also the negotiation of the readers together will be facilitated which can increase the efficiency of a tracking system. There are different scenarios for this integration including: (1) integrating tags with nodes in which sensors collect sensed information and RFID identify the objects, (2) integrating tags with sensor nodes and wireless devices in which tags can communicate with wireless devices and other tags and imitate a multiple hop network, (3) integrating RFID readers with the sensor nodes and wireless devices in which readers are able to communicate more efficiently with each other in this network and sending information to a host computer, and finally (4) integrating a separate combination of the RFID and the sensor nodes in which an integrated nodes system is not required since WSN and RFID data are processed in a software layer [48].

Various authors have investigated this integrated system for various tracking purposes by emphasizing on the indoor environment. For example, in [48] this system was used for the purpose of supply chain management. In their system, the RFID technology was employed for identification and WSN was applied for sensing the environmental factors such as humidity, temperature, and air quality since some products needed to be kept in a certain condition. In this way, tags were embedded in 
objects and readers were integrated with nodes for the purpose of identification and monitoring of the environment [27]. In another application, researchers in [26] employed a WSN for a better data transfer between RFID nodes in which they confirmed the positive contribution of WSN on communication and network flexibility. In another case study by them, they used a similar system for tracking construction operations by using tags as mobile units, which were carried by or mounted on the tracked resources. Besides, in their system, the third dimension (elevation) read from a pressure sensor [26]. In [49], also a hybrid WSN-RFID system was investigated for tracking people and objects in indoor scenarios. Through their case study, they showed that this hybridization provides an improvement of $1.6 \mathrm{~m}$ in RMSE and of $4 \%$ in data availability in a sample tracking experiment. The application of this integrated system for enhancement of safety management in a site has also been investigated by some authors. For instance, in [29] a system based on the integrated WSN and RFID technologies was employed to provide a preventive solution for enhancing the site safety. To do that, they proposed a real-time tracking system to provide pro-active information for crucial and controllable objects by attaching some RFID tags to those objects on site. A sensor node, on the other hand, transmitted sensing information to a router while the RFID tags transmit identification and positioning information to the active RFID readers. Finally, all information was sent to a central server for application rendering and alarm generation to make an early warning while a worker is too close to the hazard area [29].

The WSN offers good radio coverage but with low positioning accuracy due to high noise on received signal strength index (RSSI) measurements. On the other hand, RFID technology provides positioning information but with limited coverage and temporal discontinuity. Therefore, integration of these two technologies makes it possible to enhance the accuracy and robustness of the indoor positioning and tracking by providing better communication between RFID tags, RFID readers, and tag-readers. Having said that, some experiments have shown degraded performance of the RFID systems in object localization as active RFID tags and readers starts to get their required electrical power from WSN nodes $[26,29,48]$.

\subsubsection{RFID Integration with Laser Scanning (LS)}

The integration of laser scanning with RFID technology could be performed in different scenarios. In a typical scenario, both RFID and LS technologies are employed separately based on the type of information required from each object or activity [14,39]. For example, in [25] a model to track the state of materials on site was proposed to integrate the RFID technology with a terrestrial laser scanning (TLS). However, the two technologies were employed in two different stages through a construction supply chain system. Another scenario is an effective integration of these two technologies in which the identification and positioning capability of the RFID system help to enhance the accuracy of the point cloud data that are generated by a laser scanner [14]. Despite various techniques for object recognition from images on site, recently the process of directly extracting different types of objects from point cloud data through 3D reconstruction of building interiors is becoming more popular [54]. Besides, there are a number of advanced software solutions for 3D modeling in CAD/BIM models by using the TLS point cloud data. However, they still do not address the need for modeling all types of objects available in a room. In fact, in order to have robust point could data of the elements in a room, it is required that the room be as empty as possible when scanned. In this way, in [14,39] a methodology was proposed based on the integration of laser scanner technology with an identification technology such as RFID in order to enhance the process of object recognition out of the point cloud data. In their work, the process of extracting objects from point cloud data were not necessarily limited to structural elements (e.g., floor, ceiling, wall, windows, etc.) but also small objects and components on site were recognized, localized, and modeled in a 3D model (e.g., BIM or CAD). So, by attaching RFID tags to the objects (i.e., furniture, facilities, etc.) available on site, it was possible to identify, localize and model these components in a final 3D model too. In this scenario, the structural elements were modeled by using the TLS data. Then, the other objects were identified with RFID tags attached to them and sensed by a reader which was installed next to the laser scanner. To do that, discriminatory geometric 
information was used to recognize the target objects in a point cloud data. It was done by measuring dimensional parameters of an object (e.g., length, width, and height) or the pattern of different elements in the object. Since each object had a specific ID, it was possible to retrieve its geometric information from a facility management database and to model them more precisely in the 3D model. Saying that, in order to enhance the accuracy of the positioning algorithm, the 3D model of each piece of objects was stored in the facility management (FM) database with a mesh resolution which is automatically adopted from the point density of the acquired point cloud. This data fusion approach provided a more comprehensive and accurate 3D modeling of the as-built status of the site for the purpose of the progress reporting and productivity analysis of activities varying from structural to facility installations, etc. [14].

This integration provides an acceptable measurement accuracy by localization and angular error in the range of few centimeters and degrees respectively. Furthermore, it can facilitate the process of object recognition from the point cloud data acquired by a laser scanner. Having said that, the approach has some drawbacks such as degraded localization accuracy when the objects are in the corner of a room. Besides, it is needed to create a facility management database including all objects available in the site prior to the modeling process [14].

\subsection{Integration of the Vision-Based Technologies Together and with Other RTLS}

Using vision-based technologies such as photogrammetry, laser scanning, and their integration are appropriate choices to provide enough point cloud data in order to create 3D models of a site. More specifically, their integration enhances the point cloud data that are required for 3D reconstruction of the objects available on site. However, all these approaches have their limitations associated with their employment in a congested area and their drawbacks in modeling non-stationary and small objects such as furniture, equipment, etc. In this way, by integrating these vision-based technologies with a real-time locating systems (RTLS) technology such as robotic total station (RTS) and UWB, it is possible to overcome some of these problems. Table 4 provides a comparison between these integrated systems over the individual usage of photogrammetry or laser scanning. They are different in terms of the level of automation in data acquisition, cost, reliability, and scalability to be used in various projects.

Table 4. Capabilities of individual and integrated vision-based technologies

\begin{tabular}{cccccc}
\hline Method & $\begin{array}{c}\text { Data Acquisition } \\
\text { Effort }\end{array}$ & $\begin{array}{c}\text { Processing } \\
\text { Time }\end{array}$ & Affordability & $\begin{array}{c}\text { Data Accuracy } \\
\text { and Reliability }\end{array}$ & Scalability \\
\hline Photogrammetry & $\sqrt{ }$ & $\sqrt{ }$ & $\sqrt{ } \sqrt{ }$ & $\sqrt{ }$ & $\sqrt{ }$ \\
\hline Laser scanning & $\sqrt{ } \sqrt{ }$ & $\sqrt{ } \sqrt{ }$ & $\sqrt{ }$ & $\sqrt{ } \sqrt{\sqrt{ }}$ \\
\hline $\begin{array}{c}\text { Photogrammetry } \\
\text { laser scanning }\end{array}$ & $\sqrt{ } \sqrt{ }$ & $\sqrt{ } \sqrt{ }$ & $\sqrt{ }$ & $\sqrt{ } \sqrt{\sqrt{ }}$ & $\sqrt{\sqrt{ }}$ \\
\hline Photogrammetry + RTS & $\sqrt{ } \sqrt{ }$ & $\sqrt{ }$ & $\sqrt{ }$ & $\sqrt{ }$ & $\sqrt{ } \sqrt{ }$ \\
\hline Photogrammetry + UWB & $\sqrt{ }$ & $\sqrt{ }$ & $\sqrt{ }$ & $\sqrt{ } \sqrt{\sqrt{ }}$ \\
\hline Laser scanning + RFID & $\sqrt{ } \sqrt{ }$ & $\sqrt{ } \sqrt{ }$ & $\sqrt{ }$ & $\sqrt{ }$ & $\sqrt{ } \sqrt{ }$ \\
\hline Laser scanning + UWB & $\sqrt{ } \sqrt{ }$ & $\sqrt{ } \sqrt{ }$ & $\sqrt{ }$ &
\end{tabular}

\subsubsection{Photogrammetry Integration with Laser Scanning}

Due to the computational complexity of photogrammetric surveying, construction management researchers have attempted to decrease the number of required photos by imposing geometric constraints and automating the modeling process based on pattern recognition and feature detection [7]. Moreover, a solution based on the integration of the photogrammetry data with the data acquired by a 3D laser scanner is also investigated aiming to reduce the effort required for 3D modeling [25]. In fact, due to the geometric stability of digital images, they are very suitable references for inspection 
of the data acquired by laser scanners for 3D modeling. There are two approaches to integrating these two technologies. In one approach, the photos and scanned data are taken from the same positions, for example, the camera is installed on the laser scanner. In this approach, the extra process for coordination of different photos with scanned point cloud data is not needed, however, the photos are taken from the same distance and angle of view as scanning data which would decrease the details in photos. In another approach, photos are taken separately from scanned data but in a closer range and from different angles. Saying that in this configuration, an extra step for the orientation of the digital images with the scanned data is required [33]. This orientation can be done by choosing some common points or features in both data formats and then trying to merge them together, or by measuring the coordinates of several common points (tie points) available in both data formats in different positions $[33,50]$.

According to the literature, the new capabilities provided by this integration have brought new applications in construction management. For instance, through a case study in [50] the quantity of an excavation work accomplished was rapidly tracked for automated progress reporting. By using this integration, they proposed a more robust and timely data acquisition procedure in which fewer images and less scanning time is required to produce acceptable results during the 3D modeling process. They could also overcome limitations in photogrammetry technique associated with modeling objects with unclear geometrical shapes (e.g., excavation work) $[25,50]$. Another application for this integration is the crack detection in an object or structure. In fact, this integration helps to improve the geometry and visual quality of the final 3D model. During the data collection phase, the information about the edges and linear features in the surface such as cracks are achieved by analysis of the images, while the information about the object geometry is provided by the scanning data. In this way, a more complete 3D model of the scene can be generated with sufficient and clear details about the color, texture, and material of various objects [33,34].

The integration of a laser scanning system with photogrammetry would provide a timely, cost effective, and accurate 3D model of a structure which can decrease the human exposure costs and risk exposures caused by the lack of information during construction [38]. In fact, more accurate visual information results in less amount of rework and change order which would improve the productivity indexes [50]. Moreover, these 3D models are used for making accurate rehabilitation, maintenance, and renovation plans during the structure operation. However, the registration and alignment of these two sensory data matters. On one hand, both digital images and scanning point cloud data have internal errors that affect the registration process. On the other hand, factors such as the required accuracy, resolution and accessibility of the object may affect the chosen of the optimal method $[28,33,34]$.

\subsubsection{Photogrammetry Integration with RTS}

It is a time-dependent dynamic modeling technique in which the assets are identified by using digital imaging capabilities, while RTS units automatically provide the geo-referenced localization information for photo-based 3D modeling. In fact, each RTS unit automatically locks on a reflective glass prism which has been installed on the equipment or target object and tracks its location. Saying that, the cameras and RTS units should be placed on site with a good distance and angle of view from each other in order to enhance the quality of photogrammetry modeling and to reduce the residual error due to human factors. Besides, all cameras timer and RTS units should be time-stamped and synchronized together and being initialized in a predefined object coordinate system [7].

This integrated system makes it possible to have a dynamic visualization and tracking system for a moving object on site. In a case study [7], the sling lengths of a moving rigging system engineered for lifting heavy modules in industrial construction was tracked to ensure quality and safety. The absolute location error was estimated to be in the order of five centimeters for the measurement of the moving sling with a length of about $20 \mathrm{~m}$. 
Some newer versions of the total stations have spatial imaging capabilities with longer ranges. Moreover, by improving the machine control application, in future more integrated and synchronized actions will be done by these systems [24]. This integration enhances the 3D modeling of the moving objects by providing more accurate and reliable scaling information for the image processing-based model. However, the high cost of the RTS units and their limited capability to tack multiple moving objects are challenges for expanding their application in tracking a large number of moving objects [7].

\subsubsection{Photo/Videogrammetry Integration with UWB}

Some researchers have investigated the integration of the image-based data with UWB data in order to address problems associated with individual employment of an UWB system in a harsh and complex construction site with missing or erroneous data [3]. In fact, depending on only a single technology such as UWB to acquire required information from the site in an accurate and timely manner becomes unreliable. In this case, the employment of multiple sensors increases the reliability of the system by providing redundant and timely information as a result of either the speed of operations done by each sensor, or the parallel processing that may be achieved as part of the integration process [16]. In this integration, two important factors are scaling factor and transition factor which are used to register the point cloud data with the UWB data. Besides, there are some ways to improve the accuracy of such integration, for example by using optimization techniques, using UWB estimated positions for photogrammetric adjustment and time synchronization of the UWB and camera devices during the data acquisition process. Once the initial estimation for transformation from the photo coordinate system to UWB coordinate system is achieved, then the time-dependent camera position is corrected in order to re-estimate the transformation parameters with updated coordinates until convergence to an acceptable error [32,51].

Some potential applications of this integrated system on site include tracking of equipment and creating as-built 3D models for the purpose of activity progress reporting. This is done by improving the quality of the acquired data and providing more robust reconstruction information. Besides, it would be possible to localize the equipment even if one of these data sources is not fully available [16]. For the first application, the structure of such a system is based on the employment of the two sensory data sources in which each equipment is tagged with a UWB tag. Meanwhile, a camera is recording the operations in the monitored area. The information provided by the UWB system includes the position of the tag(s), tag update rate, the ID and type of the equipment. Imaging data, on the other hand, provides information about the position of the tracked equipment by using image processing techniques [3]. Then, the data acquired from the two sources should be integrated together. In this step, the UWB and image pixel positioning data should be aligned by transforming them from their local coordinate systems to a global coordinate system (GCS). After data association, in which the allowable error in UWB and associated image pixel positions is determined, the estimated position is calculated by averaging the position information of the UWB sensors and image processing modules. In cases, the integrated algorithm does not contain the locations of all equipment, the equipment position is estimated using the available data (e.g., UWB only or image processing only) $[3,16,51]$. In [3], a case study was implemented to measure the accuracy of this approach for resource tracking. They concluded that the UWB positioning data is better in comparison with the image processing data. However, applying image processing for more frames per second and using more advanced image processing techniques can improve the positioning accuracy of the video data.

For the second application, a UWB system is used for positioning of the camera in order to achieve information about the metric reconstruction of the site on a local coordinate system. Besides, using a $3 \mathrm{D}$ positioning technology such as UWB, a self-positioning system is achieved in which the data are directly geo-referencing (DG). It also helps to identify the parameters of the camera in a self-calibrated way which would reduce the error and computational complexity of the traditional photogrammetry techniques [32,51]. In [32], a case study was done for reconstruction of a building in which a camera and a Pozyx UWB device were mounted on an unmanned aerial vehicle (UAV) in a very 
close range from each other (less than the UWB accuracy range). In this way, first, the positioning of the receivers was done by both geo-referencing approaches included DG such as using RTS or GNSS devices, and self-positioning approach using the UWB system. The first approach was more accurate since the exact positioning of the receivers has an important role in the exactness of a proper transformation (i.e., scaling factor, translation, and rotation). After that, they achieved the position of other receivers in the same coordinate system by using trilateration and extended Kalman filter (EKF) techniques. The computed roving tag positions in the UWB coordinate system were used to estimate the transformation parameters (i.e., scaling and transition factors) for mapping the photogrammetry point clouds from the photo to the UWB frame. They concluded that introducing different weighting factors for various reading ranges, might improve the scale factor estimation. Besides, from these positioning data which will be used as reference positions, the camera external parameters can also be extracted [32,51].

The integration of these two sensing technologies provides accurate and timely positioning and visual data for various applications, especially in areas with limitation to use a positioning technology such as GNSS (e.g., in the tunnels or area covered with tall buildings) or using laser scanners due to limitation associated with scanning positions. Furthermore, there is no need for any extra devices for geo-referencing of images. In fact, in the case of direct geo-referencing using the UWB self-positioning technique, there is no need to use control points (CPs) anymore. However, the data acquired by UWB $\operatorname{tag}(\mathrm{s})$ need to get converted to the camera referenced frame and the photo coordinate system which may cause an error propagation phenomenon due to the intrinsic inaccuracy in each technology $[32,51]$.

\subsubsection{Point Cloud Data Fusion with UWB Data}

While employment of the GPS-based systems for tracking resources is an appropriate choice for an unconfined environment and outdoor tracking, the use of UWB, RFID, and wireless local area network (WLAN) seems more appropriate for indoor tracking. However, on some occasions (e.g., the blockage, congestion, metal influence, etc.) the performance of the UWB based systems could be degraded. In this case, fusion of the UWB data with a laser scanning point cloud data can help to overcome these limitations. Plus, laser scanners provide a robust solution for object recognition, visualization, and 3D modeling of a site $[6,28]$.

Integration of these two technologies, besides employment of other sources of information (e.g., 3D models, BIM, reports, etc.) have found very useful applications in construction industry. In fact, by having a better visualization model of a site and real-time information for progress reporting and schedule updating, it is possible to provide more robust and timely information for an earned value analysis (EVM) $[6,15,28]$. For instance, researchers in [15] integrated a 2D laser scanner with a UWB system in order to improve their simultaneous localization and mapping (SLAM) algorithm for localization of a moving robot. They demonstrated that this integration provides better visualization of the site due to its ability to accurately measure the range to nearby objects. Besides, this data fusion approach provided a real-time and drift-free SLAM algorithm based on ranging measurement information of the UWB system. It also helped to improve the UWB-only localization accuracy and overcoming the drawback associated with accumulated errors in scan matching needed in mapping with LiDAR. In fact, the pose estimation of a robot acquired by the UWB ranging measurements improved by subjecting it to a scan matching procedure. In [32], the results of their case study were validated for an integrated photogrammetry and UWB system with point cloud data acquired by a laser scanner to investigate the accuracy of their system for reconstruction of a building facade. In [6], a platform was proposed in which the data from this data fusion approach are integrated with other non-sensory data (e.g., reports, payments, 3D CAD, and BIM). Their platform provided more capabilities in which not only objects but also activities were tracked for real-time progress reporting on site. In fact, previous studies in this field were mainly focused on tracking objects on site and they have limitations to track non-structural activities which are not based on the tracking of a moving object, examples of such activities are welding, inspection, etc. They developed some algorithms for 
data fusion in high levels for object and activity recognition and tracking. In fact, they believed that data fusion in low levels especially in construction sites with complex working nature could be noisy, inaccurate, and incomplete due to the difference in dimensions and properties of the data acquired from various sources. In this way, trying to fuse data at higher levels decreases the computational complexity and reregistration efforts. In this way, they achieved the data for material and activity tracking by the UWB sensors and an algorithm called 3D marking, while the object recognition was done by using point cloud data acquired by a laser scanner (or photogrammetry). Besides, 3D CAD or BIM models were propped as prior information for having better object recognition. Finally, by adding administrative documents (e.g., schedule, progress reports, etc.) to the acquired information through project control applications, the EVM estimated and updated [6,28].

This integration makes it possible to detect and measure deviations from as-planned models by integrating the point cloud-based object recognition information with the UWB data for object and activity tracking on site. Besides, the UWB sensors can solve the problem of the 3D scanning in case of blockage or invisibility. However, the discrepancy between the accuracy of the UWB data and LiDAR mapping data need to be addressed $[6,28]$.

\section{Discussion on Current Solutions for Automated Data Acquisition}

Considerable studies were reported on automated data acquisition on construction sites to overcome problems associated with manual data collection. However, most of these studies were based on single sensory data acquisition $[11,16,20,23]$. Examples of these individual sensing technologies include usage of the RTLSs such as GPS, RTS, and UWB technologies for real-time tracking of resources on site. Moreover, imaging technologies such as photogrammetry and laser scanning have been widely investigated by researchers $[8,35]$. In this way, recent studies in this field are focused on integrated data acquisition technologies. However, fusing the data acquired from sensing technologies is challenging in terms of the need for manual post-processing and variation in type of data that are acquired. Therefore, having an effective data management system has an important role in technologies working based on sensory data fusion for being employed in large-scale and complex construction environment [28].

Integrated systems based on fusion of the UWB data with point cloud data (acquired by using laser scanning, photogrammetry, or their integration) make it possible to track objects and activities on site by more accuracy and reliability. In fact, it provides more reliable as-built information for construction managers for the purpose of a real-time progress reporting, earned value analysis and automatic schedule updating. Besides, using BIM models during the construction phase has found applications with the advancement in updating BIM models with as-built models [1]. In fact, the integration of these vision-based technologies with the BIM model would make it possible to modify and complete the BIM models that were created at the design level. Then, by communicating back this information to the BIM model, it would be possible to make future maintenance and management plans $[8,9,31]$. However, it is still challenging specially for facilities in which the as-built conditions differs from the as-planned models. The two vision-based technologies, photo/videogrammetry and laser scanning, have made it possible to acquire 3D as-built data for 3D modeling in a point cloud format. Saying that, the use of camera-based technologies are beneficial in terms of cost and flexibility to be used in various site condition. However, the point clouds generated in this way are not as accurate as the ones acquired by the laser scanner since the quality of photogrammetry-based point clouds is very sensitive to constraints such as camera specification, position and calibration. Plus, the accuracy of the image frames registration in photogrammetry depends on finding common points between two or more frames, so if one constraint is not respected, then the overall image quality will be affected. A laser scanner with high resolution can generate over 700 million points in one scanning session, while the point cloud density generated by photogrammetry is much less than this value $[55,56]$.

Besides, in order to make a 3D model more robust and to measure the deviation from as-planned models (e.g., placement deviation), the use of other supplementary technologies such as RFID, 
RTS, and UWB systems have been investigated by researchers. The identification and localization information provided by RFID or UWB systems are resulted in a better object reconstruction in an indoor environment. To do that, the UWB or RFID tags are attached to the subjected elements need to be tracked. In this way, it can contribute the process of object identification and extraction from the acquired point cloud data [14]. This information is used to create a better 3D as-built model of the elements associated with a specific construction activity by comparing them with as-planned models (e.g., BIM, CAD, etc.) for real-time progress reporting. Integration of the photogrammetry with the UWB technology also helps to have a direct geo-referencing system in an indoor environment with limitation to use GPS-based technologies [3,32]. Plus, by having a time-stamped location information of the digital camera, it would be possible to know from what perspective (or position) each photo frame has been taken in order to compare with the same position in the 3D models (e.g., BIM) which has applications for a more automated and real-time progress reporting [8].

Regarding the studies reviewed in this paper, improving the data collection process to acquire a complete set of as-built data with more efficiency as a project progresses and gets more complex would be an area of interest in the future. This can help to move from manual as-built modeling to an automated 3D modeling. However, investigating the use of the various RS technologies over manual techniques for automated data collection needs more attention in terms of providing economic justification for different applications. As a solution, the integration of the various RS technologies was investigated in this paper in order to address part of these issues. Suggestions for future research in this field include: (1) a more comprehensive data acquisition system in which a larger number of activities and objects are addressed, (2) improving the strategies for better fusion and registration of the sensing data acquired on site to benefit more efficiently from them. It can be done by deciding on the level of data fusion required (low to high) based on each application and the accuracy demanded, (3) deviation estimation from as-planned models to identify changes in a project more accurately and timely, (4) enhancing the compatibility and connection between these automated data acquisition technologies with the available software (e.g., Revit, Navisworks, etc.).

\section{Summery and Concluding Remarks}

Cost overrun and schedule delays are common problems, frequently leading to disputes and costly claims in the delivery of construction projects. Real-time progress reporting could address these issues by providing more timely and accurate information about activities on construction jobsites. Such timely reporting would enable decision makers and project managers to mitigate issues that give rise to the cost and schedule problems referred to above by making the schedule updating, progress reporting, and productivity analyses more frequent and accurate over project delivery period. Conventional systems for progress tracking, however, are usually manual, labor-intensive, time-consuming, and not accurate enough. In this way, automated data acquisition on site has been proposed by many researchers in order to have more robust and timely information to address the issues mentioned above. To do so, various studies have proposed the employment of different remote sensing technologies and their integrations in order to have a more automated data acquisition system. The selection of the technologies varies based on various factors such as the application, the required accuracy and the scale of a project. This study has investigated the advantageous and limitations of using these technologies by considering the applications required in construction industry.

Indoor progress tracking has also been investigated recently by some researchers. However, indoor tracking of resources and activities have some difficulties associated with a wide range of materials and structural objects that needs to be recognized. Besides, available remote sensing technologies with applications in indoor progress reporting (e.g., RFID, UWB, laser scanning, photogrammetry, etc.) have some limitations that may affect their performance in a confined area. In fact, vision-based technologies such as laser scanning and photogrammetry or their integration are good choices to generate point cloud data. However, they need post processing steps for 3D modeling which is manual and time consuming. Besides, the geo-referencing of the system during data collection 
really matters for having a more accurate data registration. In this way, the use of tracking technologies such as RTS and UWB with good localization accuracy can help to facilitate these problems and to have a more automated system for 3D modeling.

The fusion of the data acquired from various RS technologies and with other technologies has also been investigated as a solution to overcome the limitations of each technology. The activity-based approaches for progress reporting, is another area of interest in recent studies which enable us to not only track activities related to the moving objects but also to track activities which do not have such traceable objects. Finally, the registration of the data acquired by various RS technologies with available building models (e.g., 3D CAD, BIM, etc.) also needs more investigation in future.

Funding: This research received no external funding.

Conflicts of Interest: The authors declare no conflict of interest.

\section{References}

1. Omar, T.; Moncef, L.N. Data acquisition technologies for construction progress tracking. Autom. Constr. 2016, 70, 143-155. [CrossRef]

2. Su, X.; Li, S. Enhanced boundary condition-based approach for construction location sensing using RFID and RTK GPS. J. Constr. Eng. Manag. 2014, 140, 04014048. [CrossRef]

3. Siddiqui, H. UWB RTLS for Construction Equipment Localization: Experimental Performance Analysis and Fusion with Video Data. Master's Thesis, Dept. of Information Systems Engineering, Concordia University, Montréal, QC, Canada, 2014.

4. Montaser, A.; Moselhi, O. RFID indoor location identification for construction projects. Autom. Constr. 2014, 39, 167-179. [CrossRef]

5. Li, H.; Chan, G. Integrating real time positioning systems to improve blind lifting and loading crane operations. Constr. Manag. Econ. 2013, 31, 596-605. [CrossRef]

6. Shahi, A.; Safa, M. Data fusion process management for automated construction progress estimation. J. Comput. Civ. Eng. 2014, 29, 4014098. [CrossRef]

7. Siu, F.; Lu, M. Combining photogrammetry and robotic total stations to obtain dimensional measurements of temporary facilities in construction field. Vis. Eng. 2013, 1, 4. [CrossRef]

8. Kropp, C.; Koch, C. Interior construction state recognition with 4D BIM registered image sequences. Autom. Constr. 2018, 86, 11-32. [CrossRef]

9. Bosché, F.; Ahmed, M. The value of integrating Scan-to-BIM and Scan-vs-BIM techniques for construction monitoring using laser scanning and BIM: The case of cylindrical MEP components. Autom. Constr. 2015, 49, 201-213. [CrossRef]

10. Cheng, C.F.; Rashidi, A. Activity analysis of construction equipment using audio signals and support vector machines. Autom. Constr. 2017, 81, 240-253. [CrossRef]

11. Li, N.; Gerber, B.B. Performance-based evaluation of RFID-based indoor location sensing solutions for the built environment. Adv. Eng. Inform. 2011, 25, 535-546. [CrossRef]

12. Cai, H.; Andoh, A.R. A boundary condition based algorithm for locating construction site objects using RFID and GPS. Adv. Eng. Inform. 2014, 28, 455-468. [CrossRef]

13. Andoh, A.R.; Su, X.; Cai, H. A framework of RFID and GPS for tracking construction site dynamics. In Proceedings of Construction Research Congress; ASCE: West Lafayette, Indiana, 2012.

14. Valero, E.; Adán, A.; Bosché, F. Semantic 3D reconstruction of furnished interiors using laser scanning and RFID technology. J. Comput. Civ. Eng. 2015, 30, 04015053. [CrossRef]

15. Song, Y.; Guan, M.; Tay, W.P. UWB/LiDAR Fusion For Cooperative Range-Only SLAM. In Proceedings of the International Conference on Robotics and Automation (ICRA), Brisbane, Australia, 21-25 May 2018.

16. Cheng, T.; Venugopal, M. Performance evaluation of ultra wideband technology for construction resource location tracking in harsh environments. Autom. Constr. 2011, 20, 1173-1184. [CrossRef]

17. Yoo, J.; Park, J. Indoor Localization Based on Wi-Fi Received Signal Strength Indicators: Feature Extraction, Mobile Fingerprinting, and Trajectory Learning. Appl. Sci. 2019, 9, 3930. [CrossRef]

18. Kalikova, J.; Krcal, J. People counting by means of wi-fi. In Proceedings of the Smart City Symposium, Prague (SCSP), Prague, Czech Republic, 25-26 May 2017. 
19. Ta, V.C. Smartphone-based indoor positioning using Wi-Fi, inertial sensors and Bluetooth. In Machine Learning; Université Grenoble: Alpes, France, 2017.

20. Shehab, T.; Moselhi, O. An automated barcode system for tracking and control of engineering deliverables. In Construction Research Congress; ASCE: San Diego, CA, USA, 2005.

21. Ergen, E.; Akinci, B. Life-cycle data management of engineered-to-order components using radio frequency identification. Adv. Eng. Inform. 2007, 21, 356-366. [CrossRef]

22. Langley, R.B. RTK GPS. GPS World 9, 9, 70-76. Available online: https://www.gpsworld.com (accessed on 1 September 1998).

23. Labant, S.; Gergel'ová, M.; Weiss, G. Analysis of the Use of GNSS Systems in Road Construction. In Proceedings of the IEEE Geodetic Congress, Gdansk, Poland, 22-25 June 2017.

24. Hahn, P. Total Stations The Other Machine Control Sensor and More Machine Control Magazine. 2(2). Available online: http://www.machinecontrolonline.com (accessed on 1 January 2012).

25. El-Omari, S.; Moselhi, O. Integrating automated data acquisition technologies for progress reporting of construction projects. Autom. Constr. 2011, 20, 699-705. [CrossRef]

26. Ibrahim, M. Models for Efficient Automated Site Data Acquisition. Ph.D. Thesis, Dept. of Building, Civil and Environmental Engineering, Concordia University, Montréal, QC, Canada, 2015.

27. Valero, E.; Adán, A. Integration of RFID with other technologies in construction. Measurement 2016, 94, 614-620. [CrossRef]

28. Shahi, A. Activity-Based Data Fusion for Automated Progress Tracking of Construction Projects. Ph.D. Thesis, Dept. of Civil Engineering, Waterloo, ON, Canada, 2012.

29. Wu, W.; Yang, H. An integrated information management model for proactive prevention of struck-by-falling-object accidents on construction sites. Autom. Constr. 2013, 34, 67-74. [CrossRef]

30. Maneesilp, J.; Wang, C. RFID support for accurate 3D localization. IEEE Trans. Comput. 2012, 62, 1447-1459. [CrossRef]

31. Park, J.; Cho, Y.K. A BIM and UWB integrated mobile robot navigation system for indoor position tracking applications. J. Constr. Eng. Proj. Manag. 2016, 6, 30-39. [CrossRef]

32. Masiero, A.; Fissore, F. A low cost UWB based solution for direct georeferencing UAV photogrammetry. Remote Sens. 2017, 9, 414. [CrossRef]

33. Rönnholm, P.; Honkavaara, E. Integration of laser scanning and photogrammetry. Int. Arch. Photogramm. Remote Sens. Spat. Inf. Sci. 2007, 36.3/W52, 355-362.

34. Alshawabkeh, Y. Integration of Laser Scanning and Photogrammetry for Heritage Documentation. Ph.D. Thesis, Institut für Photogrammetrie, Universität Stuttgart, Stutgart, Germany, 2006.

35. Feng, Y.; Golparvar-Fard, M. Image-based localization for facilitating construction field reporting on mobile devices. Adv. Inf. Comput. Civ. Construct. Eng. 2019, 585-592.

36. Ruiz, A.; Jiménez, R. Comparing Ubisense, Bespoon, and Decawave uwb location systems: Indoor performance analysis. IEEE Trans. Instrum. Meas. 2017, 66, 2106-2117. [CrossRef]

37. Omari, S.; Moselhi, O. Integrating automated data acquisition technologies for progress reporting of construction projects. In Proceedings of the 26th International Symposium on Automation and Robotics in Construction, Austin, TX, USA, 24-27 June 2009.

38. Musa, R. BIM for Existing Buildings: A Study of Terrestrial Laser Scanning and Conventional Measurement Technique. Master's Thesis, Joint Study Programme of Metropolia UAS and HTW, Berlin, Germany, 2017.

39. Valero, E.; Adan, A. Automatic construction of 3D basic-semantic models of inhabited interiors using laser scanners and RFID sensors. Sensors 2012, 12, 5705-5724. [CrossRef]

40. Ubisense Series 7000. Available online: https://ubisense.com (accessed on 1 January 2009).

41. Bespoon SpoonPhone. Available online: http://bespoon.com (accessed on 1 January 2015).

42. Decawave TREK1000 Evaluation Kit. Available online: https://www.decawave.com/product/trek1000evaluation-kit (accessed on 1 January 2016).

43. Xu, Y.; Shmaliy, Y.S. Robust and accurate UWB-based indoor robot localization using integrated EKF/EFIR filtering. IET Radar Sonar Navig. 2018, 12, 750-756. [CrossRef]

44. Magdy, I.; Moselhi, O. Automated productivity assessment of earthmoving operations. J. Inf. Technol. Construct. (ITcon) 2014, 19, 169-184.

45. Seo, W.; Hwang, S. Precise outdoor localization with a GPS-INS integration system. Robotica 2013, 31, 371-379. [CrossRef] 
46. Jo, K.; Lee, M. Road slope aided vehicle position estimation system based on sensor fusion of GPS and automotive onboard sensors. IEEE Trans. Intell. Trans. Syst. 2016, 17, 250-263. [CrossRef]

47. Akhavian, R.; Behzadan, A.H. Construction equipment activity recognition for simulation input modeling using mobile sensors and machine learning classifiers. Adv. Eng. Inform. 2015, 29, 867-877. [CrossRef]

48. Mirshahi, S.; Uysal, S. Integration of RFID and WSN for supply chain intelligence system. In Proceedings of the International Conference on Electronics, Computers and Artificial Intelligence, Pitesti, Arkansas, Romania, 27-29 June 2013.

49. Xiong, Z.; Song, Z. Hybrid WSN and RFID indoor positioning and tracking system. Eurasip J. Embed. Syst. 2013, 1, 6. [CrossRef]

50. El-Omari, S. Automated Data Acquisition for Tracking and Control of Construction Projects. Ph.D. Thesis, Dept. of Building, Civil and Environmental Engineering, Concordia University, Montréal, QC, Canada, 2008.

51. Masiero, A.; Fissore, F. Performance Evaluation of Two Indoor Mapping Systems: Low-Cost UWB-Aided Photogrammetry and Backpack Laser Scanning. Appl. Sci. 2018, 8, 416. [CrossRef]

52. Song, L.; Tanvir, M. A cost effective material tracking and locating solution for material laydown yard. Procedia Eng. 2015, 123, 538-545. [CrossRef]

53. Skibniewski, M.J.; Jang, W.S. Localization technique for automated tracking of construction materials utilizing combined RF and ultrasound sensor interfaces. Comput. Civ. Eng. 2007, 657-664.

54. Nasrollahi, M.; Bolourian, N.; Hammad, A. Concrete surface defect detection using deep neural network based on lidar scanning. In Proceedings of the CSCE Annual Conference, Laval, Greater Montreal, QC, Canada, 12-15 June 2019.

55. Ahmed, M.; Guillemet, A.; Shahi, A.; Haas, C. Comparison of point-cloud acquisition from laser-scanning and photogrammetry based on field experimentation. In Proceedings of the CSCE 3rd International/9th Construction Specialty Conference, Ottawa, ON, Canada, 14-17 June 2011.

56. James, D.; Eckermann, J.; Belblidia, F. Point cloud data from Photogrammetry techniques to generate 3D Geometry. In Proceedings of the 23rd UK Conference of the Association for Computational Mechanics in Engineering, Swansea University, Swansea, UK, 8-10 April 2015.

(C) 2020 by the authors. Licensee MDPI, Basel, Switzerland. This article is an open access article distributed under the terms and conditions of the Creative Commons Attribution (CC BY) license (http://creativecommons.org/licenses/by/4.0/). 